\title{
Carbon Quantum Dots for Biomedical Applications: Review and Analysis
}

\author{
Nayab Azam, Murtaza Najabat Ali* and Tooba Javaid Khan \\ Prosthetics and Implantology Laboratory, Biomedical Engineering and Sciences Department, School of Mechanical and \\ Manufacturing Engineering (SMME), National University of Sciences and Technology (NUST), Islamabad, Pakistan
}

Carbon quantum dots (CQDs) are a new type of nano-carbons that are currently favored over semiconductor quantum dots (QDs) because of their solubility, low toxicity, ecofriendliness, and cheap and facile synthesis giving desired optical characteristics. Moreover, their physiochemical properties can be controlled by their synthetic route. CQDs can emit fluorescence in the range from the UV to the near-infrared (NIR) region, making them suitable for biomedical applications. Fluorescence in these nano-carbon atoms can be tuned by varying the excitation wavelength. As of now, CQDs have been used in various applications such as in bioimaging, biosensing, electrochemical biosensing, drug delivery, gene delivery, photodynamic therapy in the treatment of cancers, pharmaceutical formulations, and treating inflammation. This article highlights

OPEN ACCESS

Edited by:

Ashok K. Sundramoorthy, SRM Institute of Science and

Technology, India

Reviewed by:

Senthil Kumar $S$,

VIT University, India

Mani Govindasamy,

National Taipei University of Technology, Taiwan

*Correspondence: Murtaza Najabat All drmurtaza@smme.nust.edu.pk

Specialty section:

This article was submitted to Carbon-Based Materials,

a section of the journal

Frontiers in Materials

Received: 26 April 2021

Accepted: 02 July 2021

Published: 24 August 2021

Citation:

Azam N, Najabat Ali M and Javaid Khan T (2021) Carbon

Quantum Dots for Biomedical Applications: Review and Analysis.

Front. Mater. 8:700403.

doi: 10.3389/fmats.2021.700403 the current progress and advancement of CQDs with focus on their synthetic routes, chemical and optical properties, and biomedical applications along with new perceptions in this interesting and promising field.

Keywords: carbon quantum dots, physicochemical properties, synthetic route, fluorescence, biomedical applications

\section{INTRODUCTION}

Carbon quantum dots (CQDs), a new class of carbon nanomaterials, are bright photoluminescent quasi-spherical nanoparticles comprising amorphous to nanocrystal cores with mainly graphitic $\mathrm{sp}^{2}$ or $\mathrm{sp}^{3}$ carbon hybridization having size less than $10 \mathrm{~nm}$ (Zheng et al., 2015). Owing to their vast potential for an extensive range of technological applications especially in biomedicine, the peculiar properties of CQDs have prompted detailed studies on them. In recent years, CQDs along with their electrochemical, chemical, and excitation-dependent luminescence characteristics have drawn keen interest. Seminal advances in this research field are occurring frequently in conjunction with a number of significant breakthroughs unfolding in the last couple of years.

In 2004, Xu and his co-workers accidently discovered CQDs with the separating and purifying single-walled carbon nanotubes (SWCNTs) (Xu et al., 2004). This encouraged researchers to explore the fluorescence characteristics of quantum dots and initiate a brand new class of suitable fluorescent carbon nanomaterials. Two years later, Sun and co-workers successfully demonstrated a synthetic route to prepare CQDs with bright fluorescence emission through surface passivation and named them "carbon quantum dots" (Sun et al., 2006). There are two synthetic methods to fabricate CQDs: top-down and bottom-up approaches. The top-down approach is basically the destruction or dispersion of carbonaceous macromolecules into nano-sized CQDs by chemical, physical, or electrochemical methods, whereas in the bottom-up process, carbonaceous micro-molecules go through a series of polymerization and carbonization reactions by a chemical reaction to produce CQDs. Oxidized CQDs have many hydroxyl and carboxyl groups on their surfaces. The oxygen 


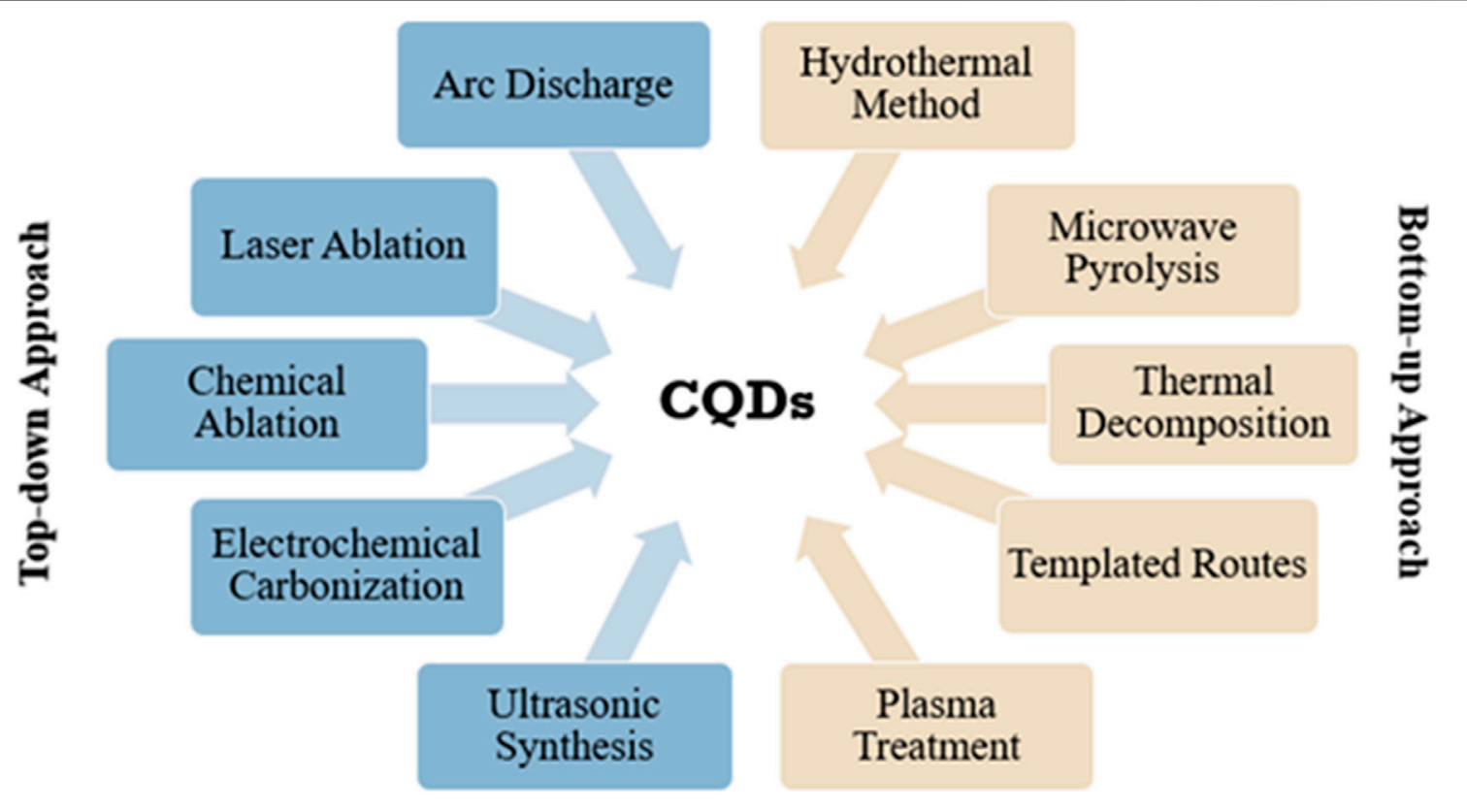

FIGURE 1 | CQD preparation by "top-down" and "bottom-up" approaches.

content varies from 5 to $50 \%$ by weight depending upon the synthetic route taken (Baker and Baker, 2010). This gives them excellent aqueous solubility and makes them able to change easily through chemical modification, surface functionalization, and surface passivation with numerous organic, inorganic, polymeric, or biological entities, allowing tuning of fluorescence and modifying physical properties. As compared to other advanced carbon nanomaterials, for instance, carbon nanotubes, nano-diamonds, fullerenes, and graphene sheets, CQDs demonstrate their significant characteristics comprising of distinct, isotropic shapes with ultrafine dimensions and modifiable surface functionalities together with facile, rapid, and cheap synthetic preparations that are appealing to a wide variety of applications such as in bioimaging (Peng et al., 2017), biosensing (Kong et al., 2012), drug delivery (Sarkar et al., 2017), tumor invasive biomarker detection ( $\mathrm{Lu}$ et al., 2016), and electrochemical biosensing (Campuzano et al., 2019), as well as sensing applications in food analysis (Pan et al., 2020).

CQDs are also a good replacement for semiconductor quantum dots even though they have been widely studied for decades due to their intense and tunable fluorescence characteristics which enable their application in the biomedical field. Nevertheless, semiconductor quantum dots have some drawbacks, such as high toxicity because of the usage of heavy metals in their preparation. It is well established that heavy metals are considered to be extremely toxic even at lower levels which makes them less suitable for clinical studies, whereas CQDs have low-toxicity, hydrophilic surface, non-blinking fluorescence, photobleaching resistance, easy passivation, chemical stability, and good cellular compatibility (Yang et al., 2014). Because of safety hazards that are both environmental and biological, CQDs are at the forefront of major research initiatives to synthesize nontoxic and environmental-friendly alternatives with desirable properties.

This article focuses on three main domains: elucidating the synthetic routes for their fabrication, chemical and optical properties of CQDs, and recent progress and development in biomedical applications and benefits they can provide to this field. Keeping in view some exceptional review articles dealing with different features of CQDs such as their physicochemical properties, fluorescence properties, synthesis routes, and biomedical applications, not only this article aims to provide a detailed outline but also the ongoing research on CQDs exposes new ideas and gives complete analysis on this amusing research field and miscellaneous scientific and technical features regarding their progress, achievements, and challenges.

\section{SYNTHETIC METHODS}

A large variety of techniques have been developed to fabricate CQDs since their discovery. Generally, synthetic methods taken for CQD preparation are divided into two routes: top-down and bottom-up methods (Figure 1). The top-down approach mainly refers to the destruction or dispersion of carbonaceous macromolecules such as graphite, nano-diamonds, carbon nanotubes, and activated carbon into nano-sized CQDs by physical, chemical, or electrochemical methods, whereas in the bottom-up process, small carbonaceous molecules such as citric acid, glucose, and sucrose go through a series of polymerization and carbonization reactions by chemical reactions to produce CQDs. 


\section{Top-Down Approaches \\ Laser Ablation}

Laser ablation is used to prepare a wide variety of nanocomposites with easily controllable morphology (Sun et al., 2006; Cao et al., 2007; Yang et al., 2009a). The ideal approach is to use a laser pulse with very high energy to irradiate the surface of target molecules and transfer it into the thermodynamic state where high temperature and pressure are created. This rapidly evaporates the target molecules and transfers it into a plasma state where they crystallize to form nanoparticles. $\mathrm{Li}$ and his co-workers demonstrated a simple approach to synthesize CQDs from nano-carbon particles dispersed in different solvents, using laser irradiation ( $\mathrm{Li}$ et al., 2010). The formulated CQDs exhibited stable, observable, and tunable PL. Moreover, $\mathrm{Hu}$ and his coworkers established that the CQD surface can be modified by choosing a suitable organic solvent to tune the PL properties of the prepared CQDs (Hu et al., 2009). Subsequently, later, it was discovered that the size of CQDs can be altered by changing the width of the laser pulse which can affect CQDs' growth and nucleation process ( $\mathrm{Hu}$ et al., 2011). CQDs that passivated PEG200 were synthesized by laser irradiation system using a continuous flow jet (Doñate-Buendia et al., 2018). A higher number of CQDs were produced in smaller sizes as compared to batch processing. Consequently, this provided a better opportunity for large-scale production of CQDs. CQDs produced were applied in in vitro human cell lines for imaging. Recently, the double-pulse femtosecond laser ablation method was used to produce CQDs where the size reached a minimum value of $\sim 1 \mathrm{~nm}$ depending on the delay in the pulses. Therefore, the mean sizes of CQDs obtained through double-pulse ablation are smaller than those obtained in singlepulse ablation (Figures 2A-D), and abundant functional groups are created on the surface of CQDs that can be used in catalytic and sensing applications (Nguyen et al., 2020). Laser ablation is an effective method to prepare CQDs with good aqueous solubility, narrow size range, and fluorescence characteristics. However, it is a complex process and expensive which is why its applications are limited.

\section{Chemical Ablation}

Chemical ablation is a process in which small organic molecules are carbonized to nano-carbonaceous materials with strong oxidizing acids, which are further drawn into small sheets by a controlled oxidation (Ray et al., 2009; Tian et al., 2009). There is no need of any complicated apparatus for the synthesis of CQDs which can be produced at a large scale. However, harsh conditions and drastic processes are the major drawbacks of this procedure. Peng and his co-workers demonstrated a simple route for luminescent CQD synthesis by using concentrated sulfuric acid for the dehydration of carbohydrates (Tian et al., 2009). The obtained carbonaceous material was broken into separate CQDs by nitric acid treatment, followed by passivation with amine-terminated compounds. Passivation of the surface was vital for the luminescence of CQDs. The emitting wavelength of CQDs can be altered by treating the starting material with nitric acid with different time durations. The multicolor emission and biocompatibility of obtained CQDs enable them to be applied in biomedical sciences. PL CQDs were prepared by carbonization and functionalization of polyethylenimine (PEI) used as a carbon source as well as by passivation in nitric acid treatment. Generally, CQDs are insensitive to $\mathrm{pH}$, but CQDs achieved from PEI were sensitive to $\mathrm{pH}$ (Shen et al., 2013). Hence, these characteristics strongly support that they can used as proton sensors in observing the biological process through proton release. In vitro results suggested that the obtained CQDs can easily enter the cell membrane; therefore, they can be used as a biolabeling agent in living cells.

\section{Electrochemical Carbonization}

Electrochemical carbonization is a simple and convenient technique for synthesizing CQDs from bulk of carbon precursors under normal temperature and pressure conditions (Anwar et al., 2019). It is a widely reported method since it is easy to tune the PL and size of the synthesized CQDs. Zhang and his co-workers prepared CQDs through this method by using low-molecular-weight alcohols (Deng et al., 2014). A traditional three-electrode system was used where two Pt sheets and a reference electrode were used. The obtained CQDs exhibited bright fluorescence without any complicated passivation and purification procedures. The applied potential can be used to control the size of CQDs. Thus, different small molecular alcohols can be used to produce high-QY CQDs that are less toxic to human cancer cells, which ultimately can be applied in bioimaging. In 2018, N-CQDs emitting green fluorescence were prepared using the same three-electrode system utilizing pyrocatechol and ethylenediamine which were precursors and electrolytes (Niu et al., 2018). The ON-OFF fluorescence principle was used to detect the presence of pyrophosphate anions (PPi) and alkaline phosphatase (ALP) by allowing $\mathrm{Fe}^{3+}$ to quench fluorescence of N-CQDs. This provided rapid, simple, low-cost, and high-sensitivity detectors that have an enticing opportunity in in vivo and in vitro clinical diagnosis as well as promoting the use of nano-carbons in biosensors.

\section{Ultrasonic Synthesis}

The ultrasonic sound is renowned to generate high and low pressure alternatively in the liquid to form and destruct small vacuum bubbles. Cavitation produced in these bubbles can cause speeding of liquid jets, de-agglomeration, and robust hydrodynamic shear forces. Hence, the robust energy of ultrasonic waves can break down large carbon materials into smaller CQDs (Li H. et al., 2012; Park et al., 2014). An optical nano-probe was devised to detect methylmercury through the fluorescence assay (Costas-Mora et al., 2014). D-Fructose (carbon source) and PEG for surface passivation were given ultrasonic treatment to synthesize CQDs. The presence of the target analyte, during the synthesis, increases the potential of CQDs as fluorescent probes. The as-prepared CQDs were about $2.5 \mathrm{~nm}$ which are suitable for a portable fluorospectrometer in analysis. Dang and his coworkers synthesized CQDs from an oligomer polyamide 

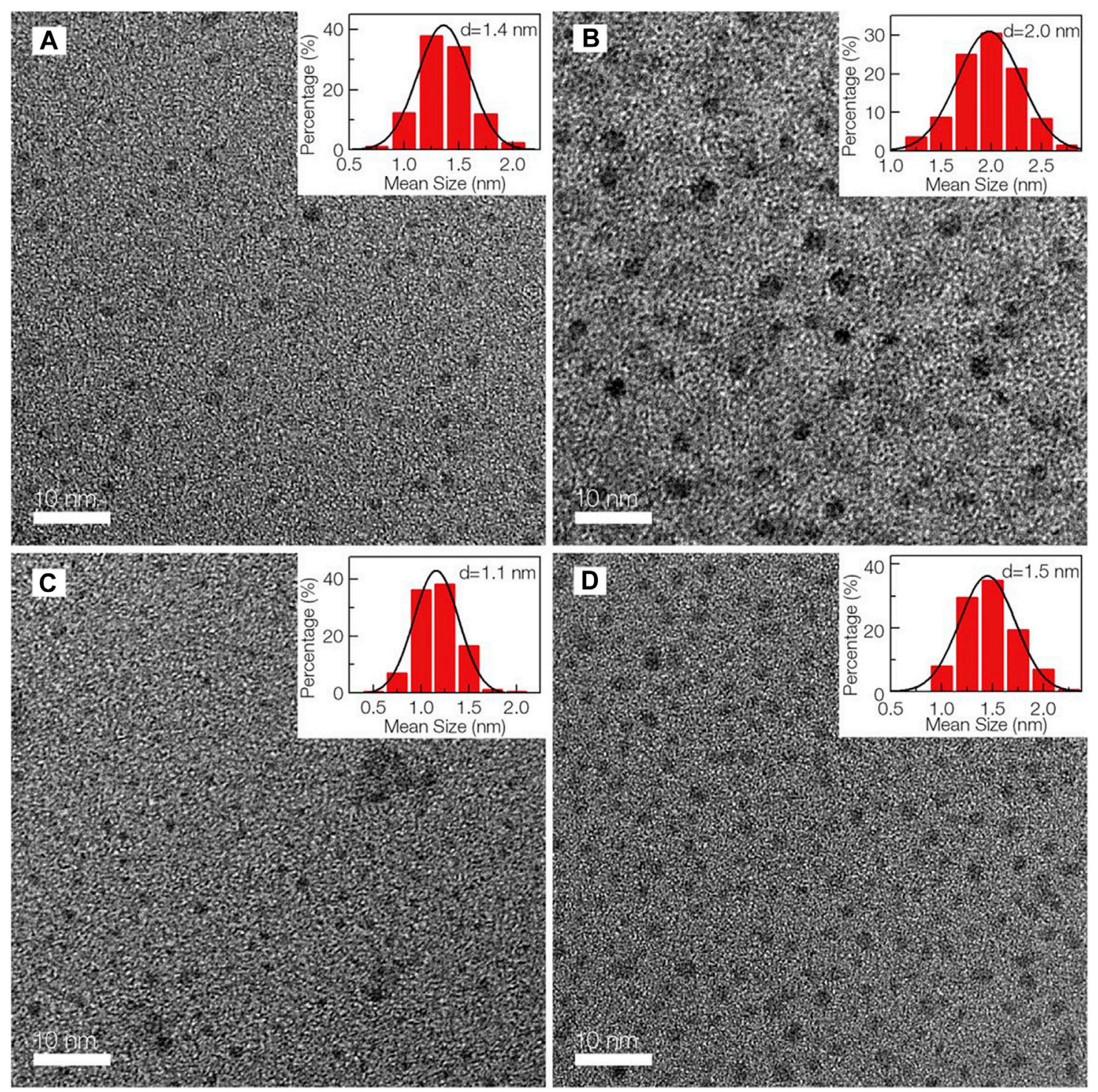

FIGURE 2 | TEM images of CQDs and their subsequent size distributions. Adapted from Nguyen et al. (2020).

resin via the ultrasound method (Dang et al., 2016). CQDs produced showed low crystallinity, good dispersion, and surface-rich functional groups.

\section{Bottom-Up Approaches Hydrothermal Method}

Hydrothermal carbonization (HTC) is one of the most commonly used methods to fabricate novel carbonic nanomaterials from a variety of primary raw materials because it is usually a one-step process which is cheap, is environmentally friendly, has low resistance to photobleaching, and is non-toxic; it produces CQDs nearly of uniform size with high luminescence (Titirici and Antonietti, 2010). The standard way is to dissolve organic molecules in a solvent and transfer to a Teflon-lined stainless- steel reactor. The reaction precursors are fused together at relatively high temperature for a few hours to form carbon cores that eventually grow into CQDs with a particle diameter less than $10 \mathrm{~nm}$. Zhu and co-workers stated the maximum QY of CQDs reached up to $80 \%$ which was equal to that of fluorescent dyes (Zhu et al., 2013). CQDs formulated from citric acid as a carbon source and ethylene diamine as a nitrogen source via the hydrothermal process were used to detect $\mathrm{Fe}^{3+}$ in living cells. Efficient CQDs were produced using rice and glycine as carbon and nitrogen sources by a facile hydrothermal method (Qi et al., 2019). These CQDs were used as a probe which detected $\mathrm{Fe}^{3+}$ and tetracycline (TC) antibiotics remarkably. Hola and his colleagues synthesized controllable fluorescent full-color CQDs by varying the amount of nitrogen under hydrothermal decomposition of 
citric acid and urea (Hola et al., 2017). Furthermore, biomolecules rich in carbon and nitrogen can be used to tune the lattice structure of CQDs under the hydrothermal process by varying the temperature, producing $89.57 \%$ of QY (Lu et al., 2019). Simplicity and capability of controlled nitrogen doping of CQDs made this method to be used in the field of bioimaging and biosensors.

\section{Microwave Pyrolysis}

The microwave, an electromagnetic wave, has wide spectra of wavelengths ranging from 1 to $1,000 \mathrm{~mm}$ that gives robust energy to break down the chemical bonds present in a substrate. So, the microwave method can efficiently reduce the time duration of the reaction and give immediate, homogeneous heating, to produce uniform CQDs. The rapid synthesis and commercialization of this method make it preferable in bottom-up approaches. Recently, it is discovered that microwave pyrolysis of specific small molecules in liquid media can successfully formulate CQDs of bright fluorescence and aqueous solubility (Choi et al., 2017). Lysine was used to produce such CQDs in less than 5 min of microwave treatment where thermal polyamidation followed by carbonization reaction occurred to create lysine-based CQDs. The results showed that they were highly non-toxic and biocompatible and can be used for bioimaging of cellular media safely. The selection of appropriate monomers and regulating the polymerization reaction during microwave treatment are extremely crucial for successful development of highly soluble and intense PL CQDs. Liu and co-workers produced highly luminescent CQDs by using citric acid and several amine molecules in microwave pyrolysis (Zhai et al., 2012). The primary amine molecules played a double role as $\mathrm{N}$-doping and surface passivating agents for preparing CQDs that helped in tuning the PL intensity. As the N-content increased, the QY values elevated for the CQDs up to $30.2 \%$. The results depicted the high biocompatibility of CQDs that have great potential in the biomedical field.

\section{Thermal Decomposition}

Thermal decomposition is a method where external heat is provided to dehydrate and carbonize organic materials to form CQDs. This process is inexpensive, does not necessarily require solvent, and is easy to operate in a scalable operation (Zhai et al., 2012). Wang and co-workers synthesized highly fluorescent $(\mathrm{QY}=47 \%)$ CQDs from citric acid as the carbon source and organosilane as the passivation agent at high temperature for $1 \mathrm{~min}$ and produced CQDs of $0.9 \mathrm{~nm}$ (Wang F. et al., 2011). These non-water-stable CQDs can easily be transformed into water-soluble CQDs that have low toxicity to selected cell lines. Fluorescent CQDs were simply fabricated by heating ascorbic acid in aqueous media at low temperature (Jia et al., 2012). The results showed that CQDs possessed favorable biocompatibility, excellent aqueous dispersibility, and pH-, excitation-, and polarity-dependent luminescence and up-conversion fluorescence properties. These simply synthesized CQDs can serve as a future candidate for fluorescence probes, biosensors, and bioimaging devices.

\section{CHARACTERIZATION}

Carbon in its elemental form is a black mineral and is known to have poor fluorescence and less soluble in water. However, CQDs have received a great deal of attention for exhibiting high luminescence and good solubility and are thus also called carbon nano-lights. Carbon-based quantum dots procure various properties depending on their structure. Functional groups such as the carboxyl on the CQD surface introduce biocompatibility and water solubility. Numerous biological, polymeric, organic, or inorganic materials are used for surface passivation and chemical modification of CQDs. Surface passivation conveys different defects on the surface, hence improving the fluorescence properties of CQDs. They have stable fluorescence, conductivity, and high quantum yield (QY).

The chemical structure of CQDs depends on their synthesis. CQDs are spherical with diameter not more than $10 \mathrm{~nm}$ and are divided either into amorphous or into crystalline nanoparticles. The gap between CQD layers is $0.34 \mathrm{~nm}$ which is consistent with the (002) spacing of the crystalline graphite (Bao et al., 2011) and is distinguishable by high-resolution transmission electron microscopy (HR-TEM). In contrast, graphene quantum dots (G-CQDs) own good crystallinity with a crystal lattice spacing of about $0.24 \mathrm{~nm}$ conforming to (100) in-plane lattice boundaries of graphene (Bao et al., 2011). X-ray diffraction (XRD) is another essential technique for structural analysis of CQDs. XRD gives the information of the dimensions of the unit cell and the lattice spacing present in the crystalline carbon cores. Figure 3 shows the XRD pattern, where the diffraction peak is around $20^{\circ}$ representing the graphitic structure of the crystal and lattice spacing of about $0.45 \mathrm{~nm}$ which is more than $0.34 \mathrm{~nm}$, therefore reflecting certain amorphous characteristics in synthesized CQDs (Wang and $\mathrm{Hu}, 2014$ ). Different chemical functional groups such as carboxyl and hydroxyl groups at the surface of CQDs can be assessed through matrix-assisted laser desorption ionization time-of-flight (MALDI-TOF).

Raman scattering can determine the structural features of CQDs. Figure 4 shows a typical Raman spectrum of CQDs having two peaks representing $\mathrm{D}$ and $\mathrm{G}$ bands, respectively.

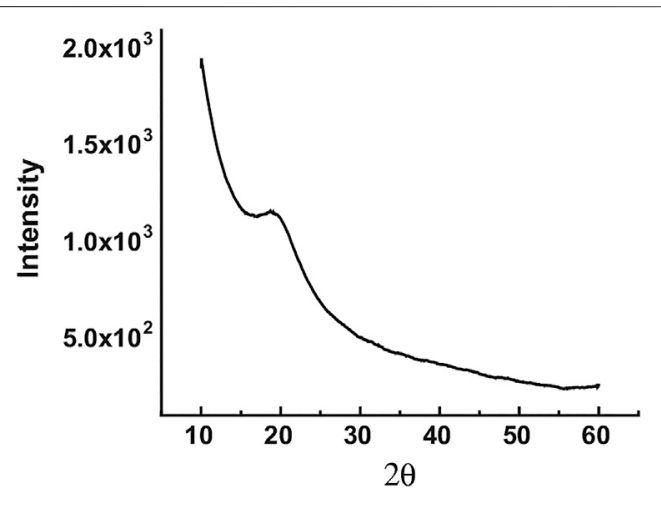

FIGURE 3 | XRD pattern of a CQD sample. A weak yet broad peak is seen around $20^{\circ}$ corresponding to a lattice spacing of about $0.45 \mathrm{~nm}$ and reflecting poor crystallinity. Adapted from Wang and Hu (2014). 


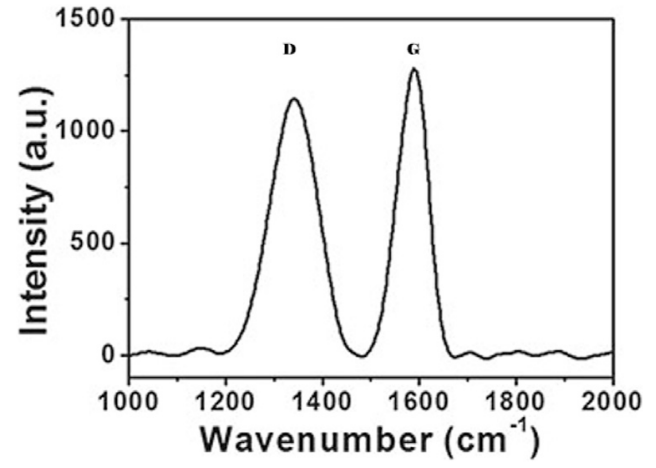

FIGURE 4 | Raman spectrum of the CQD sample. D and G bands are represented at 1,350 and $1,600 \mathrm{~cm}^{-1}$, respectively. The IG/ID ratio in this case is 1.12, indicating more abundant graphitic carbon atoms (Wang and $\mathrm{Hu}$, 2014).

The $\mathrm{D}$ band $\left(1350 \mathrm{~cm}^{-1}\right)$ corresponds to disordered $\mathrm{Sp}^{2}$ carbon atoms, and the $\mathrm{G}$ band $\left(1600 \mathrm{~cm}^{-1}\right)$ corresponds to the in-plane stretching vibration mode $\mathrm{E} 2 \mathrm{~g}$ of crystalline graphite carbon atoms. The ID/IG ratio of intensities of Raman bands is used to study the carbon framework especially the degree of crystallinity and relative abundance of carbon atoms as compared to surface atoms. X-ray photoelectron spectroscopy (XPS) helps in identifying specific atomic units present on the surface of CQDs. Fourier-transform infrared (FTIR) spectroscopy also provides information on functional units present in the sample by recording typical vibration bands (Figure 5). In bottom-up methods, CQDs are fabricated from poly-aromatic hydrocarbons through dehydration and carbonization via the hydrothermal method, microwave pyrolysis, and the plasma-hydrothermal method (Wang and $\mathrm{Hu}, 2014)$. All of these methods can easily regulate the characteristics of the final product.

\section{PROPERTIES OF CARBON QUANTUM DOTS}

\section{Optical Properties}

CQDs retain most remarkable and unique properties such as a wide spectrum of light emitting from ultraviolet (UV) to near infrared (NIR), tunable fluorescence, efficient multiphoton upconversion and down-conversion, and all of the characteristics that can be effectively modified through the size, shape, surface functionalization, and surface doping on the basis of quantum confinement effect (QCE), surface effect, and edge effect.

\section{Absorbance}

CQDs usually display absorption of light from the UV region $(260-320 \mathrm{~nm})$ to the visible range $(400-700 \mathrm{~nm})$. CQDs are photon-harvesting agents which is why absorption is maximum in short wavelength. The highest peak is around $230 \mathrm{~nm}$ which is attributed to the $\pi-\pi^{*}$ transition of $\mathrm{sp}^{2}$ conjugated carbon, whereas a shoulder around $300 \mathrm{~nm}$ is allocated to $n-\pi^{*}$ conversion of hybridization with

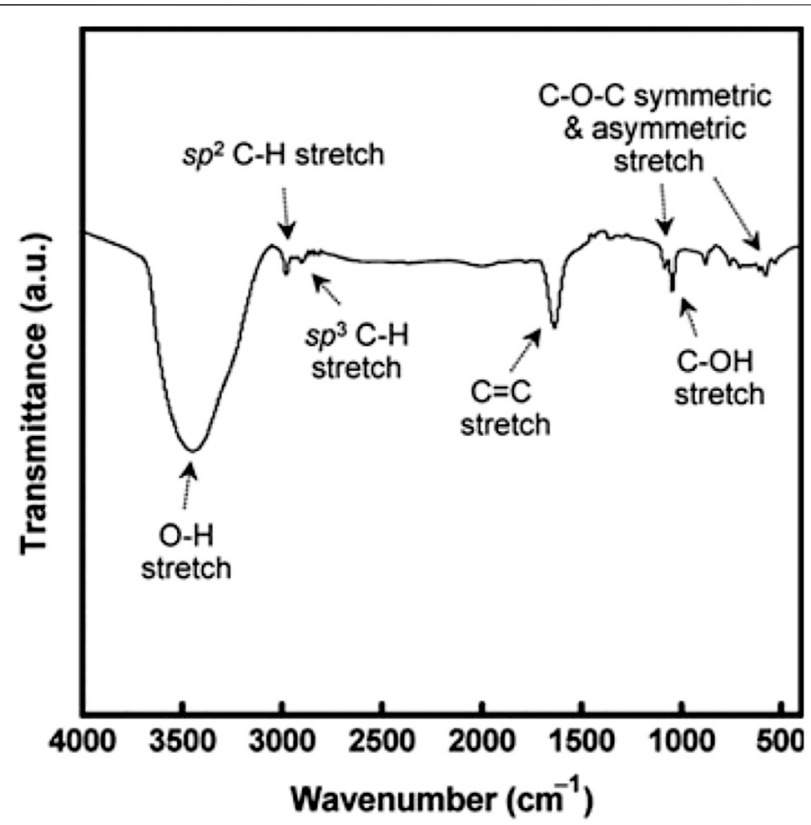

FIGURE 5 | Fourier-transform infrared (FTIR) spectrum of the CQD sample. Distinct vibration bands corresponding to CQDs' surface units are shown (Wang and Hu, 2014).

heteroatoms (for example, $\mathrm{N}, \mathrm{S}, \mathrm{Mg}, \mathrm{P}, \mathrm{O}$ ) (Lin and Zhang, 2012). Figure 6 demonstrates the absorption peaks of $\pi-\pi^{\star}$ and $\mathrm{n}-\pi *$ (Emam et al., 2017). The absorption property of CQDs can indeed be tuned by the surface passivation or modification process (Dimos, 2016). Jiang and co-workers successfully synthesized red, green, and blue luminescent CQDs via the hydrothermal method by employing three isomers of phenylenediamines (Bao et al., 2011). The gradual red-shifted absorption was observed in these three CQDs. Furthermore, heteroatom doping is an effective technique to modify the CQD absorption. Qu and his co-workers reported that the doping of CQDs with $\mathrm{S}, \mathrm{N}$ heteroatoms shifted the absorption band into the visible region 550-595 $\mathrm{nm}$ (Qu et al., 2013).

\section{Photoluminescence}

Tunable photoluminescence is the most striking property of CQDs giving them a wide spectral range and high-intensity emission peaks ranging from the ultraviolet to the visible to the NIR region. This key feature can also be referred to as excitation-dependent luminescence spectra. Hence, the emission color of CQDs is tunable according to the provided excitation wavelength using the quantum confinement effect (QCE) and size of nanoparticles (Molaei, 2020). Figure 7 demonstrates the excitation-dependent luminescence spectra of a CQD sample and their corresponding colors (Wang et al., 2019b). The PL property of CQDs originates from the energy states correlated with surface defects upon the surface passivation of CQDs for high quantum yield (QY). Many studies have demonstrated the experiments which support the luminescence model shown in Figure 8. This explains that the surface state of CQDs is directly related to luminescence. 


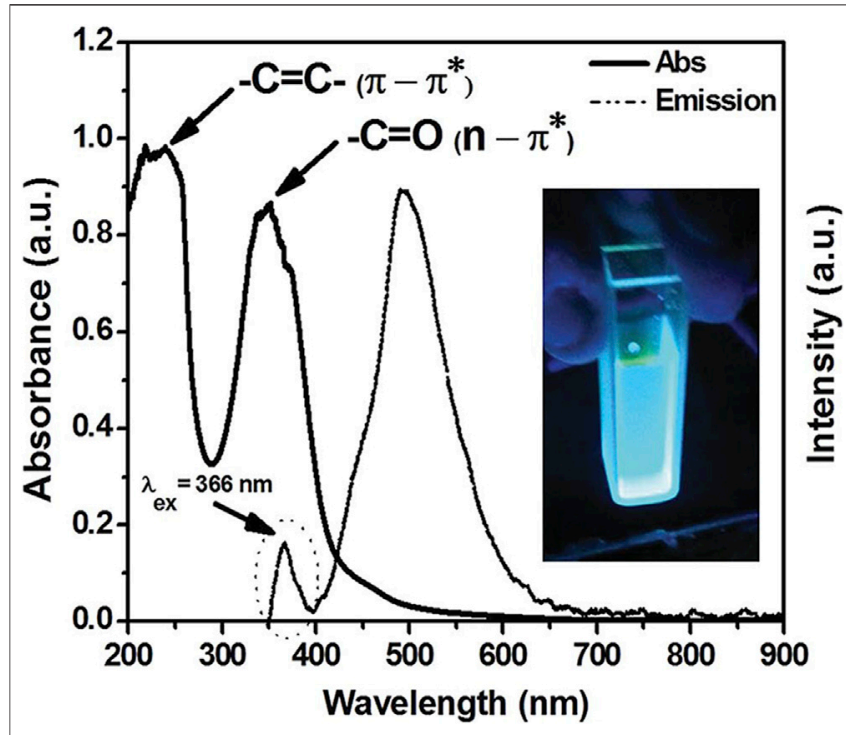

FIGURE 6 | UV-Vis absorbance spectrum of the CQD sample. A wide absorbance peak in the presence of heteroatoms is represented upon the surface of CQDs. Adapted from Emam et al. (2017).

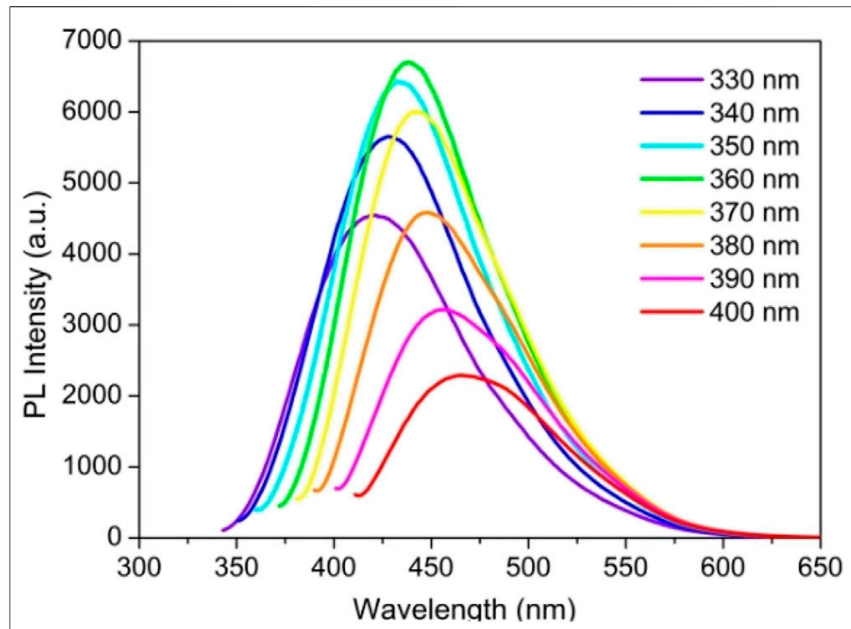

FIGURE 7 | Excitation-dependent luminescence spectra of CQDs. Adapted from Wang et al. (2019c).

However, there are several other studies that have demonstrated CQDs showing only a single emission wavelength by carefully modifying the CQD surface in such a way that it reduces surface defects that are responsible for different energy levels. In order to control the excitationdependent emission profiles of CQDs, H. Lin and his colleagues synthesized CQDs that emitted similar peak intensities after being excited by different wavelengths (Pan et al., 2015). Another key feature of CQDs that holds is their non-blinking PL and exceptional photostability when compared with those of organic fluorophores. The non-blinking PL characteristic allows tracking of a single molecule, whereas

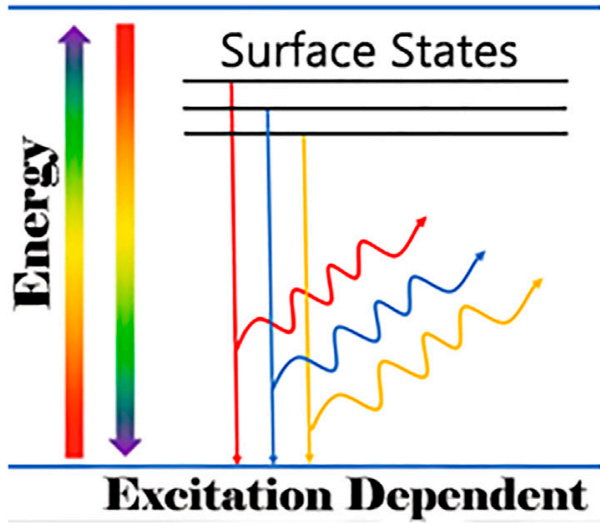

FIGURE 8 | Multicolor photoluminescence of CQDs attributed to different surface energy states. Adapted from Wang and Hu (2014).

photostability enables long-term imaging in real time. The intensity of PL CQDs synthesized by the laser ablation method is decreased by only $4.5 \%$ after $4 \mathrm{~h}$ as compared to that of organic fluorophores that photobleached within $0.5 \mathrm{~h}$ (Li et al., 2010). Several experiments have shown CQDs sensitive to $\mathrm{pH}$ affecting their photostability.

\section{Chemical Luminescence}

$\mathrm{CL}$ is the phenomenon which involves the excitation of CQDs by a chemical reaction. The redox reaction is responsible for creating a number of electron holes in CQDs which accelerate electron hole annihilation leading to the release of energy in the form of $\mathrm{CL}$ emission. The intensity of CL depends on the occurrence and yield of chemical reaction. In the standard CQD CL mechanism, two reactions occur in parallel in a solution. One is responsible for the electrons to transfer to the excited energy level called electron injection, whereas the other reaction scavenges electrons from the lower energy level of CQDs called hole injection. As a result, CL occurs when injected electrons transfer from the excited energy level to the lower energy level. This mechanism was reported in the study by L. Zhao that the CQDs emitted intense CL in a strong alkaline solution of $\mathrm{NaOH}$. $\mathrm{NaOH}$ stimulated the reduction of residues at the surface of CQDs which showed that CQDs donate electrons well to the oxygen atom dissolved in a solution to form a superoxide anion radical in the solution. The combined effect of injected electrons through chemical reduction and the thermally created cavity results in strong luminescence (Zhao et al., 2013). CQDs acting as both an electron donor and an acceptor hold great opportunities in photocatalysis, but their application is still limited because of a complex redox reaction and coupling to CQDs.

\section{Electrochemiluminescence}

CQDs have been studied for the electrochemical luminescence (ECL) property as well which occurs when electrochemically induced electrons and holes trigger excited states in CQDs and then relax to their ground states when light is emitted (Zheng et al., 2009). Generally, it is accepted that luminescence occurs 


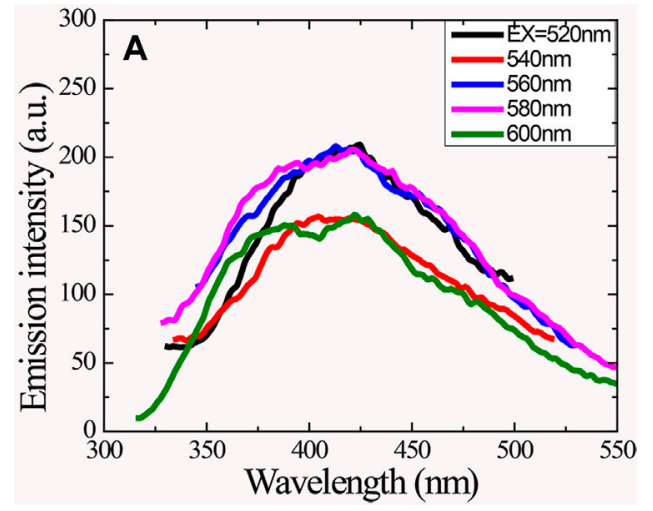

FIGURE 9 | UCPL emission of CQDs excited at different wavelengths. Adapted from Gude (2014).

when surface states excite electrons and emit light when they relax to their lower energy state. But here, shorter wavelength luminescence in CQDs is assigned to core energy levels of CQDs for great energy separation rather than surface states. Henceforth, this topic is still under debate.

To comprehend further how PL and ECL are affected by the configuration, shape, and surface structure of CQDs, two types of CQDs were formulated having CQDs with low oxidation level (r-CQDs) and high oxidation level (o-CQDs) through a carbonization-extraction strategy and carbonization-oxidation process, respectively (Li L.-L. et al., 2012). The findings revealed that the distribution of o-CQDs $/ \mathrm{KS}_{2} \mathrm{O}_{8}$ onto the electrode surface causes the electrochemical response. The "loose shell" having oxygen-containing groups on the o-CQDs generates o-CQD radicals, the oxidizing agents. The $\mathrm{SO}_{4}{ }^{-}$radical is released when $\mathrm{S}_{2} \mathrm{O}_{8}{ }^{2-}$ accepts an electron from anionic o-CQD ${ }^{-}$for ECL emission. The ECL wave was initiated at $1.10 \mathrm{~V}$, and it reached its maximum peak at $1.30 \mathrm{~V}$ which coincided with the oxidation peak of cyclic voltammograms (CVs). Therefore, the ECL emission is directly related to the oxidization of o-CQDs. A light-emitting diode (LED) device made from CQDs was reported which has current-density-dependent emission. Different ECL from the same CQD sample generated light from blue to white range under different voltages (Zhang et al., 2013).

\section{Up-Conversion Photoluminescence}

CQDs also show the UCPL property where two or longer wavelength photons are activated through the simultaneous absorption and emit luminescence of shorter wavelength than excitation wavelength. This fascinating optical property accounts for many applications in the field of cell imaging with two-photon luminescence microscopy and photocatalysis from the visible to the NIR region. Cao and co-workers first exhibited CQDs that produced $5 \mathrm{~nm}$ of wavelength of visible emission by a two-photon excitation mechanism in the NIR region $(800 \mathrm{~nm})$ via femtosecond pulsed laser ablation (Cao et al., 2007).

Later on, other researchers also observed UCPL emissions from CQDs that were fabricated from different methods. For instance, Kang and his co-workers discovered that CQDs prepared from ultrasonic treatment possess normal PL emission as well as UCPL properties (Li et al., 2011a). Figure 9 presents the UCPL spectrum of such CQDs which are excited from 500 to $600 \mathrm{~nm}$ wavelength and emit luminescence from 350 to $500 \mathrm{~nm}$ range, and the highest emission peak is observed at $425 \mathrm{~nm}$ (Gude, 2014). However, a few related sources may have some reservations about the UCPL. Wen and co-workers suggested that UCPL actually originates from the normal fluorescence when excitation light is diffracted and produces another second light of wavelength $\lambda / 2$ which coexists with the first one (Wen et al., 2014). Long pass can be added in the fluorescence spectrophotometer to avoid this. Essentially, UCPL is a standard fluorescence rather than the multiphoton process and can only be detected with a femtosecond pulse laser. In contrast, Shen and co-workers debated that the multiphoton process is not enough for describing up-converted luminescence of CQDs. So, they formulated GQDs from graphene oxide (GO) and passivated with polyethylene glycol (PEG). They postulated that shortwavelength UCPL emission occurs when low-energy photons excite $\pi$ electrons to the LUMO followed by falling back into the $\sigma$ orbital (HOMO). The consistent energy difference between excitation and emission light is known as the energy gap between LUMOs and HUMOs (Shen et al., 2011).

\section{Photoinduced Electron Transfer Properties}

Extensive investigations have been conducted recently on CQDs for photoinduced charge separation, electron transition processes, and photo-response for the efficient usage of PL entities in light energy conversion and other associated applications, along with mechanistic elucidation. Wang and co-workers first proposed that CQDs are both electrons donors and acceptors which is why they can be quenched efficiently by either electron acceptors like nitrotoluene and dinitrotoluene or electron donors like N,N-diethylaniline. Different electron-accepting characteristics of CQDs were observed between nitrotoluene and dinitrotoluene by observing the quenching of fluorescence intensity and lifetime. The researchers suggested that discrete quenching profiles for efficient PL in CQDs might be ascribed to the disruption of the excited state in redox reactions due to doped metals that mainly emit bright fluorescence (Wang et al., 2009). In another study, the mechanism of photoluminescence (PL) quenching of CQDs is presented using $\mathrm{Cu}^{2+}$, and it was observed that $\mathrm{PL}$ quenching of CQDs happened due to the PET process as $\mathrm{Cu}^{2+}$ in the $d$ orbit combined with the carboxyl group at the surface of photoexcited CQDs. This study not only provides the opportunity to develop sensors based on CQDs but also provides a new way for electron and energy conversion processes (Liu et al., 2018).

\section{Biological Properties}

Remarkable advancements have been made in the synthesis of bio-probes with strong PL and stable CQDs. Nevertheless, biocompatibility of CQDs is still a concern for any further applications in living cells and tissues. CQDs have been 
TABLE 1 | Synthesis of CQDs for various biomedical applications.

\begin{tabular}{|c|c|c|c|c|c|}
\hline Biomedical applications & CQDs & $\begin{array}{l}\text { Methods of } \\
\text { CQD synthesis }\end{array}$ & $\begin{array}{l}\text { Excitation } \\
\text { wavelength }\end{array}$ & $\begin{array}{c}\text { Emission } \\
\text { wavelength }\end{array}$ & Reference \\
\hline \multirow[t]{4}{*}{ Bioimaging } & PEG-CQDs & Laser ablation & $800-880$ nm & $455-790 \mathrm{~nm}$ & Yang et al. (2009) \\
\hline & PCD & Hydrothermal method & 335 nm & 460 nm & $\begin{array}{l}\text { Shereema et al. } \\
(2015)\end{array}$ \\
\hline & L-CQDs & Hydrothermal method & $375-460 \mathrm{~nm}$ & $454-535 \mathrm{~nm}$ & Xue et al. (2019) \\
\hline & $\begin{array}{l}\text { Poly(propionyl ethylenimine-co- } \\
\text { ethylenimine) CQDs }\end{array}$ & $\begin{array}{l}\text { Microwave-assisted } \\
\text { pyrolysis }\end{array}$ & 800 nm & - & Cao et al. (2007) \\
\hline \multirow[t]{2}{*}{ Biosensors } & CQD-TPY & Electrochemical method & $800 \mathrm{~nm}$ & $\sim 498 \mathrm{~nm}$ & Kong et al. (2012) \\
\hline & CDs/silica & Pyrolysis & $340-400 \mathrm{~nm}$ & $450 \mathrm{~nm}$ & Liu et al. (2014b) \\
\hline Drug delivery & T-tags (DOX-conjugated CQDs) & Ultrasonic synthesis & $350-610$ nm & $300-500$ nm & Lee et al. (2014) \\
\hline \multirow[t]{4}{*}{ Gene delivery } & Cationic CDs/pSOX9 & $\begin{array}{l}\text { Microwave-assisted } \\
\text { pyrolysis }\end{array}$ & $365 \mathrm{~nm}$ & - & Cao et al. (2018) \\
\hline & fc-rPEI-CQDs & Microwave pyrolysis & $360 \mathrm{~nm}$ & $460 \mathrm{~nm}$ & Wu et al. (2016) \\
\hline & PCD/DNA & Hydrothermal method & $335 \mathrm{~nm}$ & $460 \mathrm{~nm}$ & Hu et al. (2014) \\
\hline & CQDs-PEl & $\begin{array}{l}\text { Microwave-assisted } \\
\text { pyrolysis }\end{array}$ & $350 \mathrm{~nm}$ & 365 nm & Liu et al. (2012) \\
\hline Photodynamic therapy & SWCNTs-PEG-Fe3O4@CQDs & Hydrothermal method & $420-480$ nm & $330-390$ nm & Zhang et al. (2018) \\
\hline Photothermal therapy & FeN@CQDs & Hydrothermal method & $410 \mathrm{~nm}$ & $470 \mathrm{~nm}$ & Zhang et al. (2017) \\
\hline \multirow[t]{3}{*}{ Electrochemical biosensors } & $\mathrm{CQDs} / \mathrm{H}_{2} \mathrm{O}_{2}$ & Hydrothermal method & $340 \mathrm{~nm}$ & $440 \mathrm{~nm}$ & Barati et al. (2015) \\
\hline & PtNPs-CDs/IL-GO & Hydrothermal method & $350 \mathrm{~nm}$ & $462 \mathrm{~nm}$ & Chen et al. (2018) \\
\hline & CQDs/GQDs & Hydrothermal method & $260-320$ nm & $440-625$ nm & $\begin{array}{l}\text { Dave and Gomes } \\
\text { (2019) }\end{array}$ \\
\hline \multirow{4}{*}{$\begin{array}{l}\text { Antibacterial/antiviral effects for treating } \\
\text { cancer or inflammation }\end{array}$} & CQDPAs & Pyrolysis & $270 \mathrm{~nm}$ & $300-450 \mathrm{~nm}$ & Jian et al. (2017) \\
\hline & Quaternary ammonium CQDs & Hydrothermal method & $430 \mathrm{~nm}$ & $497 \mathrm{~nm}$ & Zhao et al. (2020) \\
\hline & NCQDs & Hydrothermal method & 335 nm & $455 \mathrm{~nm}$ & Zhao et al. (2019) \\
\hline & Cur-CQDs & Pyrolysis & $365 \mathrm{~nm}$ & $\approx 450 \mathrm{~nm}$ & Lin et al. (2019) \\
\hline \multirow[t]{2}{*}{ Pharmaceutical formulations } & Water-soluble fluorescent CQDs & Top-down approach & $360-540$ nm & $500-580 \mathrm{~nm}$ & Li et al. (2015) \\
\hline & NHF-CDs & Pyrolysis & $370-410 \mathrm{~nm}$ & $424 \mathrm{~nm}$ & Savin et al. (2019) \\
\hline
\end{tabular}

evaluated for cytotoxicity in last few years. CQDs were prepared from the arc-discharge method using graphite rods refluxed in $\mathrm{HNO}_{3}$ for $12 \mathrm{~h}$ for the cytotoxicity assay. Till the $0.4 \mathrm{mg}$ $\mathrm{ML}^{-1}$ concentration, CQDs were not toxic (Yang et al., 2009a). Zhao and his co-workers established a facile method to synthesize CQDs via electrochemical treatment of graphite that did not damage the human kidney cell line in the MTT assay (Zhao et al., 2008). The CQDs passivated with functional groups, such as PEG (Yang et al., 2009a), PEI (Wang et al., 2011b), and PAA (polyacrylic acid) (Wang et al., 2011c), were tested for cytotoxicity assays. CQDs passivated with PEG were not toxic up to a very high concentration than the required concentration needed for bioimaging and other associated applications (Yang et al., 2009b; Wang et al., 2010). Also, CQDs passivated with PEG1500N were injected to mice for 28 days and were evaluated for toxicity. The results suggested that there were no significant toxic effects, and hence, passivated CQDs were biocompatible (Yang et al., 2009b).

In another experiment, the MTT assay showed that a PEI sample was not toxic to HT-29 cells even at high concentrations, whereas the PEI-CQDs were more toxic to cells than PPEI-EICQDs because of the presence of more EI units in PEI (Cao et al., 2007). Moreover, it was discovered that free PAA in nonaqueous solution is detrimental to cells even at concentration as negligible as $50 \mathrm{mg} \mathrm{ml}^{-1}$. Both the PAA-CQDs and free PAA were compared at the same concentrations, and both were found out to be lethal to cells when exposed for $24 \mathrm{~h}$ but less toxic when they were exposed for $4 \mathrm{~h}$ (Wang et al., 2011b). In conclusion, PEG and PPEI-EI are suitable for functionalization even at high concentrations for in vivo bioimaging and biosensing. Molecules like PAA with high toxicity can still be used for CQD functionalization if their concentration and incubation period is reduced enough. To assess the cellular toxicity of N-doped CQDs (N-CQDs), the cell viability assay was conducted. N-CQDs and CQDs were incubated with HepG2 cells at different concentrations for $24 \mathrm{~h}$. The toxicity of N-CQDs was less than that of CQDs. The results showed the cell viability remained great at high concentrations, signifying the non-toxicity and good cellular compatibility of the N-doped CQDs. Thus, N-doped CQDs can be used in cell imaging (Qi et al., 2019).

\section{BIOMEDICAL APPLICATIONS}

The physicochemical and optical properties of CQDs make them an excellent candidate for biomedical applications. Their small size and cellular compatibility allow them to be used in bioimaging, biosensing, and drug delivery vehicle that can be tracked in the body using their fluorescence property. CQDs can be applied in various applications in the biomedical field such as gene delivery, electrochemical biosensing, pharmaceutical formulations, photodynamic and photothermal therapy, and treatment of bacterial infections and inflammations due to aforementioned properties of CQDs such as low toxicity, aqueous solubility, 
chemically inert, non-blinking, and excitation- and size-dependent PL emission. Some of them are discussed below with recent advances and summarized in Table $\mathbf{1 .}$

\section{Bioimaging}

QDs are also widely studied for in vitro and in vivo bioimaging in the past decades. In a number of applications, traditional QDs are preferred over organic dyes and fluorophores due to high fluorescence, stability, and resistance to metabolic degradation (Zheng et al., 2015). However, they are toxic due to the presence of heavy metals and are temperature dependent that no quantum dots could internalize at $4^{\circ} \mathrm{C}$. QDs were able to penetrate the cell through endocytosis but could not reach the nucleus. Therefore, considerable attention has been given to CQD applications in biological labeling and bioimaging. The present finding that CQDs can exhibit PL emission in the NIR spectral region is of particular interest for in vivo nanotechnology because of body tissue transparency in the NIR window.

CQDs were synthesized using carbon soot with nitric acid treatment and passivated with PEG1500, to compare with commercially available semiconductor $\mathrm{CdSe} / \mathrm{ZnS}$ quantum dots for cytotoxicity and bioimaging (Yang et al., 2009b). The results showed that CQDs are more biocompatible and luminescent with a QY of $20 \%$ than CdSe/ZnS QDs. The CQDs displayed size less than $10 \mathrm{~nm}$, whereas the QD size is more than $20 \mathrm{~nm}$. The smaller size gives advantage to CQDs to be used as probes in small biological structures, and minimum volume can be used in in vivo injections. Cao and co-workers prepared CQDs using poly(propionyl ethylenimine-coethylenimine) for surface passivation which produced high luminescence intensity with a two-photon excitation mechanism in the NIR region. The brightness was dependent on the power of a pulsed infrared laser. The passivated CQDs were compared with the semiconductor QDs reported in the literature. The aqueous soluble CQDs had size less than $5 \mathrm{~nm}$. These CQDs were incubated with human breast cancer cells, and images were taken, when excited with $800 \mathrm{~nm}$ laser pulses. CQDs labeled the cell membrane and cytoplasm but failed to reach the nucleus. To be able to label the nucleus, CQDs coupled with TAT (a human immune deficiency virus-derived protein) which allows CQDs to accumulate in cells and reach the nucleus (Cao et al., 2007).

Surface passivation allows CQDs to change their optical properties for bioimaging. In an experiment, passivated CQDs were synthesized from maltose via microwave treatment. CQDs were able to enter the cells and emit green fluorescence, and cell viability remained unaffected even after $24 \mathrm{~h}$ of incubation (Shereema et al., 2015). CQDs were also synthesized of size less than $10 \mathrm{~nm}$ by using different molar ratios of alkali lignin, citric acid, and ethylenediamine via a simple hydrothermal method. They displayed $90 \%$ cell viability, when incubated with $\mathrm{HeLa}$ cells at the concentration of $0.62 \mathrm{mg} \mathrm{ml}^{-1}$, even after $24 \mathrm{~h}$. It was observed that a large amount of CQDs entered the cells through the process of endocytosis and emitted fluorescence. They mainly accumulated in the cytoplasm, and weak emissions were observed from the nucleus. When these CQDs were excited at different wavelengths, they emitted red, green, and blue fluorescence. Due to their low toxicity and biocompatibility as well as the intrinsic ability to emit multicolor fluorescence, CQDs bring great opportunity in bioimaging and biolabeling fields (Xue et al., 2019a).

CQDs produced by the hydrothermal method have also been applied in in vivo studies for optical bioimaging. CQDs of size ranging from 2 to $6 \mathrm{~nm}$ were successfully prepared by using a biorefinery by-product (glucose and xylose), amino-passivated with urea. The photophysical properties of as-prepared CQDs were characterized to investigate their suitability for bioimaging. The QY was approximately $13 \%$ when excited with the wavelength of $365 \mathrm{~nm}$ and showed stable fluorescence behavior as compared to the commercially available fluorophore 4',6-diamidino-2-phenylindole (DAPI), even under changing temperatures from $4^{\circ} \mathrm{C}$ to $60^{\circ} \mathrm{C}$ and when undergoing photobleaching treatment. CQDs were incubated with L929 fibroblasts, and their cell viability remained greater than $90 \%$ up to $400 \mathrm{mg} / \mathrm{ml}$. For in vivo optical imaging studies, a nude mouse was inoculated with tumor cells and intravenous injection of CQDs was given to the mouse through the tail vein. Figure 10 shows images taken from 0 to $24 \mathrm{~h}$. The mouse was euthanized, and images of organs were taken as well. It was observed that both in vitro and in vivo imaging experiments showed low toxicity, antioxidant activity, and remarkable bioimaging performance of CQDs (Huang et al., 2019).

\section{Biosensors}

CQDs are also used as biosensors of their aqueous solubility, nontoxic nature, excellent cellular compatibility, excitationdependent multicolor emission, permeability to cells, high photostability, and low toxicity. The CQD-based biosensors can visually monitor glucose (Shi et al., 2011), phosphate (Zhao et al., 2011), nucleic acid (Li et al., 2011c), cellular iron (Zhu et al., 2012), potassium iron, and pH (Wei et al., 2012). CQDs are used as an excellent fluorescent assay to detect nucleic acid with selective single-base mismatch. CQDs adsorb via $\mathrm{p}-\mathrm{p}$ interactions to label a single-stranded DNA (ssDNA) probe, accompanied by fluorescence quenching and hybridizing to its target to form double-stranded DNA (dsDNA), resulting in desorption of dsDNA from the CQD surface with more recovered fluorescence, hence probing the target DNA (Li et al., 2011c). Reactive oxygen species (ROS) are important biomarkers for many diseases including cancer, DNA damage, infections or inflammations, arthritis, neurodegenerative disorders, and chemotherapeutic drug screening. Manipulation of reactive oxygen species (ROS) using oxidation-reduction active substances is a means to kill cancer cells selectively. ROS sensors have been developed to detect reactive oxygen species (ROS) using encapsulated CQDs in hydrogel made of ascorbic acid. They are used to check the efficacy of the drug by measuring ROS levels after administering chemotherapeutic compounds (Bhattacharya et al., 2017). N-doped CQDs synthesized from the one-pot pyrolysis method with a high QY of about $49.5 \%$ and high photoluminescence are used in the detection of $\beta$-glucuronidase (GLU) inhibitor activity based 

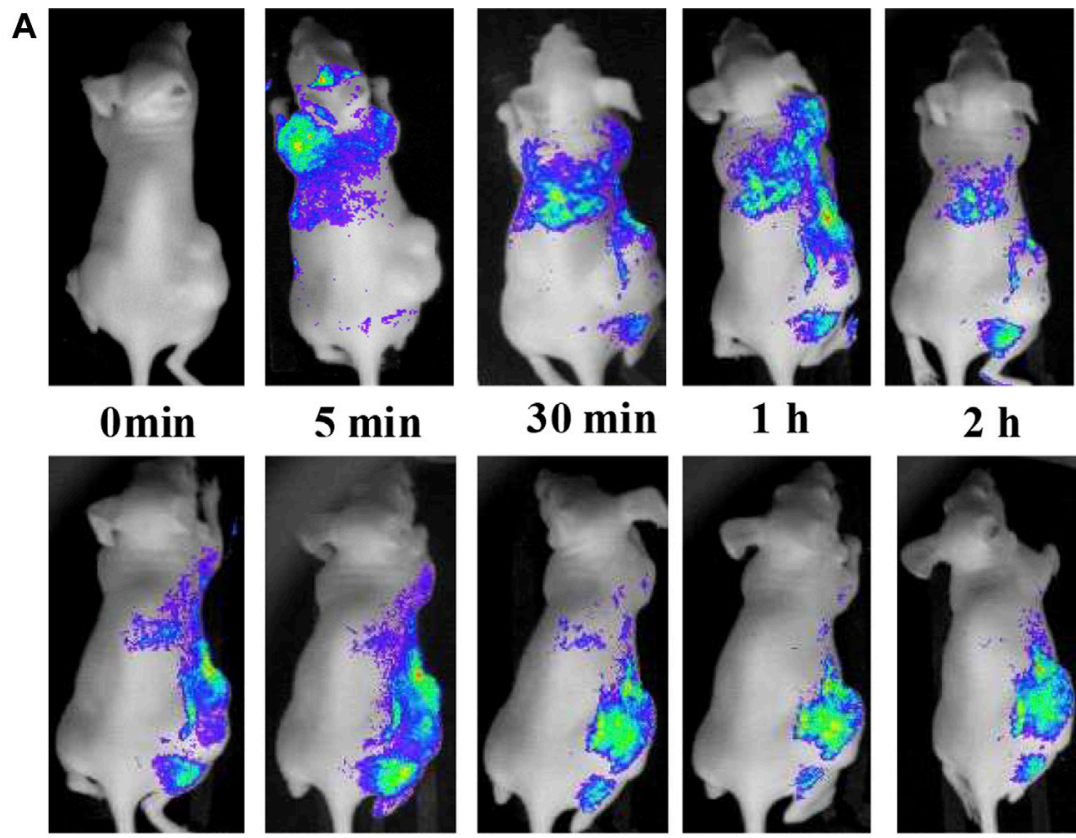

30 min

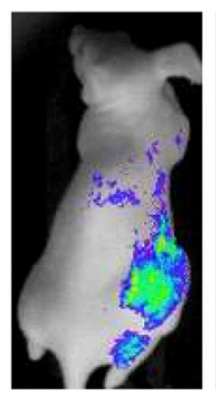

B

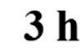

$4 \mathrm{~h}$
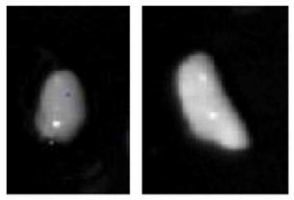

Heart

Lung

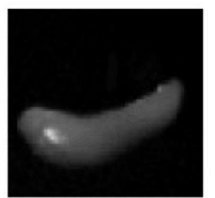

Spleen
8 h

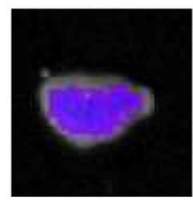

Kidney
$1 \mathrm{~h}$

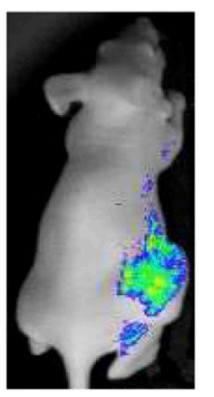

$12 \mathrm{~h}$

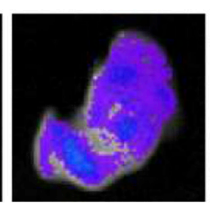

Liver
$2 \mathrm{~h}$

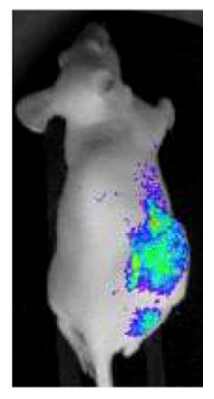

$24 \mathrm{~h}$

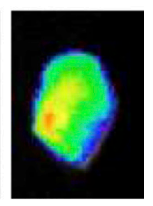

Tumor

FIGURE 10 | (A) In vivo fluorescence imaging of nude mice after IV injection of CQD solution; (B) fluorescence images of dissected organs of a mouse after IV injection of CQD solution for 24 h. Adapted from Huang et al. (2019).

on the inner filter effect (IFE). GLU used as a biomarker in earlystage cancer diagnosis and many physiological diseases has a great importance as a biomarker because of its inhibition activity to control the proliferation of cancer cells with the ability to change the fluorescence intensity of N-doped CQDs (Lu et al., 2016).

CQDs synthesized by the electrochemical method of average size $6 \mathrm{~nm}$ were used to develop a two-photon fluorescent probe for observing $\mathrm{pH}$ changes from 6 to 8.5 with high sensitivity and specificity. The described probe was effectively used in twophoton biosensing and bioimaging to monitor the $\mathrm{pH}$ changes in living cells and tissues. Kong and his colleagues demonstrated that the terpyridine-based receptor molecule (TPY) covalently binds to the CQD surface and induces change in fluorescence intensity due to adsorption-desorption of $\mathrm{H}^{+}$ions. The quantum yield of CQDs was approximately $10 \%$ and showed CQDs possess UCPL characteristics from UV to NIR spectra. The CQD-TPY nano-probe shows high selectivity toward $\mathrm{H}^{+}$ ions as compared to other metal atoms, biomolecules, and amino acids, as the $\mathrm{H}^{+}$ion concentration increases and the emission intensity also increases in the $440-650 \mathrm{~nm}$ range. The imaging and biosensing of $\mathrm{pH}$ in real time were successfully accomplished in in vitro lung cancer A549 cells and mouse LLC-MK2 cells and in vivo tumor tissues by inducing tumor cells in nude mice through two-photon microscopy (Kong et al., 2012).

So far, multiple studies have shown that CQDs fabricated by facile methods can show selective and sensitive detection of various ions, metals, and molecules. The ability of CQDs to detect metal ions is the inevitable sensing application including biological and chemical scenarios. This is due to attraction and alignment of metal ions with aromatic residues present on the surface of CQDs. Even at low concentrations when metal binds to CQDs, they cause luminescence quenching; hence, they are an effective vehicle for metal sensing. CQDs were prepared from $\mathrm{N}$-( $\beta$-aminoethyl)- $\gamma$-aminopropyl methyldimethoxysilane (AEAPMS), and the residues ethylenediamine and methoxysilane groups were on the surface to detect $\mathrm{Cu}^{2+}$ ions and were coated on rhodamine B-doped silica nanoparticles (Liu et al., 2014a). Under single excitation wavelength, rhodamine B emitted red fluorescence and CQDs emitted blue fluorescence. When CQDs interacted with $\mathrm{Cu}^{2+}$ ions, they quenched fluorescence without affecting rhodamine $\mathrm{B}$. The in vitro test was conducted to investigate 
the feasibility of dual emission nanoparticles by incubating them with MCF-7 cells. Blue and red wavelengths were emitted, and fluorescence images were taken. When $\mathrm{Cu}^{2+}$ ions were added, fluorescence intensity of blue decreased more as compared to that of red. All these findings suggest that CQDs can act as noninvasive detectors that are inexpensive and sensitive and help in improving human health.

\section{Electrochemical Biosensors}

CQDs have high stability, surface functionalization, and good electrical conductivity and exhibit great potential to be used in biosensing, optical sensing, photovoltaics, chemical sensors, nano-medicine, and electrocatalysis (Li W. et al., 2020; Wang D. M. et al., 2019). CQDs have attracted great attention in the development of electrochemical biosensors to detect biomolecules including glucose and dopamine, DNA, hemoglobin, cholesterol, glucose, L-cysteine, ovalbumin, cholines, ascorbic acid, histene, and human carcinoembryonic antigen and are involved in the detection and reduction of $\mathrm{H}_{2} \mathrm{O}_{2}$ as well as in the reduction of $\mathrm{O}_{2}$ because of their electrocatalytic properties. CQDs with polymers have also been reported in the detection of hemoglobin having brilliant sensitivity and increased selectivity (Barati et al., 2015; Kour et al., 2020). Only one example of CQDs has been reported yet to be used as an electrode modifier in the synthesis of the DNA sensor to detect single gene mutations (Campuzano et al., 2019). In one study, it has been observed that CQD nano-composites (PtNPsCDs/IL-GO) are used for the detection of $\mathrm{H}_{2} \mathrm{O}_{2}$. Due to their unique chemical structure, they are responsible for enhanced catalysis of $\mathrm{H}_{2} \mathrm{O}_{2}$ as well as providing multiple active sites for electrochemical oxidation and reduction reactions. The results of the study showed these electrochemical sensors (PtNPs-CDs/IL$\mathrm{GO})$ have a large selectivity ranging from 1 to $900 \mu \mathrm{M}$ and low detection limit of $0.1 \mu \mathrm{M}$ with respect to the reduction of $\mathrm{H}_{2} \mathrm{O}_{2}$ which are the characteristics of good electrochemical sensors having high conductivity and electrocatalytic activity (Chen et al., 2018).

Another study showed CQDs can be used in electrochemical biosensors by encapsulating CQDs in $\mathrm{Pt}$ nanocrystals and combining them with $\mathrm{N}$-doped GQDs. This nano-composite shows good catalytic activity in electrochemical biosensing of damaged DNA markers. One more electrochemical biosensor has been developed to identify glucose with glucose oxidase/GQDs/ carbon-based ceramic electrode. These graphene-based CQDs have high biocompatibility, a hydrophobic carbon frame, hydrophilic edges, and more absorption of the enzymes on electrodes which make them promising in electrochemical biosensors (Dave and Gomes, 2019).

\section{Food Toxin Detection}

As CQDs are having unique optical properties, they are being used in the detection of toxic chemicals, heavy metals, and pathogens in food (Huang et al., 2019). Natural food products are used to synthesize CQDs and to detect food toxins such as CQDs extracted from apple juice to detect $P$. aeruginosa and $M$. tuberculosis bacteria and fungal $M$. oryzae cells (Mehta et al., 2015). Heavy metals in soil cannot be degraded by microorganisms, can be absorbed by plants, enter the food products, and cause toxicity to humans, and their detection is an important concern. CQDs made of honey, corn flour, spoiled milk, and chine grass are used as fluorescent probes for imaging of $\mathrm{Fe}^{2+}, \mathrm{Cu}^{2+}, \mathrm{Cr}^{6+}$, and $\mathrm{Hg}^{2+}$ metal ions, respectively (Fan et al., 2020).

In one study, Citrus sinensis and Citrus limon peels were used to fabricate two types of CQDs using the one-pot synthesis method. Many characteristics of fabricated CQDs were the same, but there were some individual critical properties that make them different. Their strong PL properties with the presence of oxygen and nitrogen functional groups on the surface of CQDs make them able to be used in different applications. Synthesized CQDs were used as chemosensors for the detection of $\mathrm{Fe}^{3+}$ and tartrazine (Chatzimitakos et al., 2017). In another study, a sensing platform was developed based on the characteristics of fluorescence of CQDs to detect food additives. CQDs were synthesized from a leguminous plant known as flamboyant mirim (FM) as this is a cost-effective and environmentally friendly sensing technique. After their spectroscopy evaluation, PL response of CQDs was checked by LDA in the presence of citric acid, lactic acid, ascorbic acid, potassium sorbate, and sodium benzoate; these substances are used in the formulation of food products, and then FM-CQDs are used as imaging probes in the detection of additives in pickled olives (Carneiro et al., 2021). So, it can be concluded that CQDs synthesized from natural sources have potential to be used in the detection of food toxins including food pathogens, heavy metals, and toxic chemicals.

\section{Drug Delivery}

While cancer and other diseases are being conventionally treated by chemotherapy, such a route typically lacks precision and causes problems of toxicity and multi-drug resistance ( $\mathrm{Li} \mathrm{X}$. et al., 2014). Therefore, targeted drug delivery is preferred as an alternative method to improve drug bioavailability and efficacy and reduce side effects (Sharma et al., 2006; Rosenholm et al., 2010; Liu et al., 2012). But it also causes the drug to leak before it reaches the target site (Zhou et al., 2014). Hence, efficient target agents like CQDs are required as they have been proposed as dual nano-carrier systems comprising of bioimaging and specific drug delivery agents with minimum cytotoxicity.

CQDs modified through functionalization avoid cytotoxicity through drug leakage to normal cells. Functionalized CQDs contain amino groups on their surfaces which are used to crosslink with tumor theranostics. CQDs prepared from citric acid through microwave pyrolysis were crosslinked with PEGylated oxidized alginate and then conjugated with theranostic doxorubicin (DOX) through acid-labile Schiff base linkage. The in vitro test showed that theranostic nanoparticles had the ability to release drugs without any burst release in the acidic microenvironment of the tumor in a $\mathrm{pH}$-dependent manner. Moreover, their fluorescence property provided imaging-guided drug delivery (Jia et al., 2016).

CQDs have also been used for targeted delivery in the nucleus and mitochondria (Wu et al., 2017). Mitochondria play a vital role in many cancers and metabolic disorders. CQDs produced 

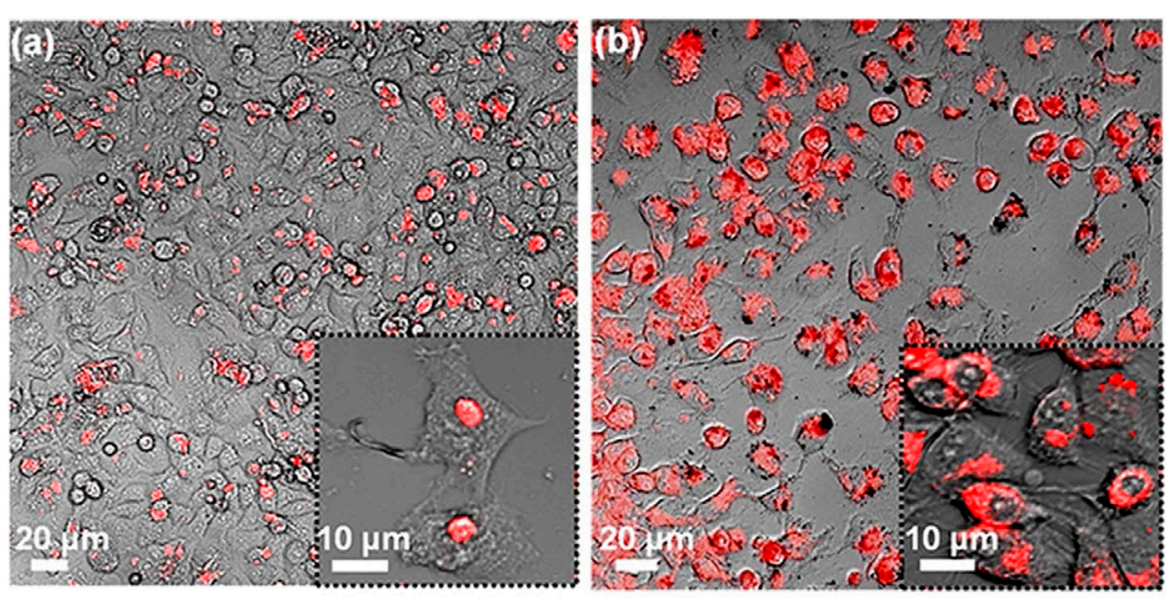

(c)

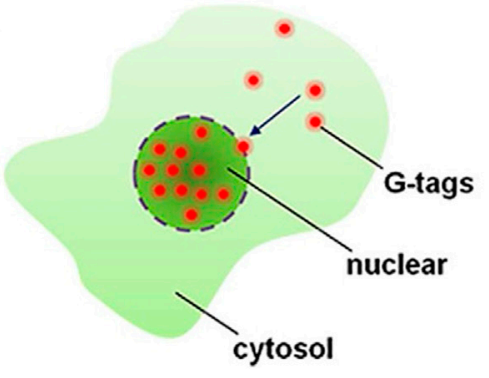

(d)

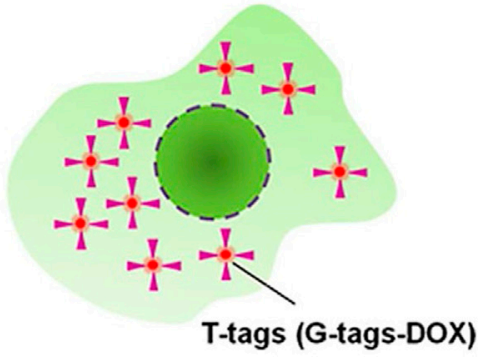

FIGURE 11 | Images were taken by confocal microscopy of the cancer cells that were incubated for 24 h. (A) G-tags (CQDs) did not cause any cytotoxic effect on cancer cells, while (B) the DOX-conjugated CQDs (T-tags) did cause cytotoxic effect on cells. (C) G-tags in the nucleus of the cells. (D) DOX-conjugated CQDs in the cytosol of the cell. Adapted from Lee et al. (2014).

from chitosan, ethylenediamine, and mercaptosuccinic acid via hydrothermal treatment were found to possess long-term mitochondrial imaging as compared to commercially available MitoTracker dye and mitochondrial-specific temperaturedependent transport and receptor-mediated endocytosis (Hua et al., 2017).

Fluorescence of CQDs and location of their emission help to differentiate the living and apoptotic cancer cells. CQDs incorporated in hydrogel were taken up by A549 cells and emitted green light when observed under fluorescence microscopy. The emission of green light depends upon the concentration of CQDs in the gel. Hydrothermally synthesized CQDs have been used in the drug delivery system to deliver the anticancer drug (DOX) to the targeted area (Molaei, 2019). In another study, it was observed that drug-loaded CQDs were more localized as compared to DOX alone. To check their activity in the body, conjugated CQDs were incubated in HepG2 and MCF-7 cells (Figure 11). It was observed that DOX-conjugated CQDs were more cytotoxic in cancer cells as compared to normal cells (Lee et al., 2014).

It is challenging to treat brain tumors and neurodegenerative disorders to partial impermeability of the brain and short retention of the drug in the brain and blood-brain barrier (Soroceanu et al., 1998). Therefore, for accurate diagnosis and prognosis, precise tumor cell imaging is required. CQDs have also been used in conjunction with tumor-penetrating peptides to target brain tumors. RGERPPR-penetrating peptides with CQDs were used to penetrate vascular walls of the tumor (Yang et al., 2013). CQDs prepared from citric acid via hydrothermal treatment were functionalized using maleimide-polyethylene glycol-amino succinimide succinate (Mal-PEG-NHS) and RGERPPR. The resultant nanoparticles successfully targeted glioma and delivered in vivo bioimaging (Gao et al., 2018).

\section{Crossing Blood-Brain Barrier}

Over the last few decades, studies on nanoparticles are more focused on the development of drug delivery vehicles. These carriers have been investigated in nano-medicine; they not only help in the formulation of drugs but also provide the sustained release of drugs at the targeted sites. For the enhanced delivery of drugs, these nanoparticles are combined with diagnostic probes and designed to target sites which are hard to reach ( $\mathrm{Xu}$ et al., 2013). Nanoparticles are important in biomedical applications because of their small sizes as the blood-brain barrier prevents crossing of drugs with large sizes such as proteins, chemicals, and peptides. To pass drugs from the blood-brain barrier, nanoparticles and CQDs can be used to deliver drugs in targeted sites, but it is essential to keep the sizes of these nanoparticles less than the size of the capillaries for use in the living body. These nano-sized particles stop the blockage of blood vessels and eliminate them from the body by the reticuloendothelial system. In brain tumors, the delivery of 
imaging probes to the tumor is difficult because of the blood-brain barrier. It is dependent upon the size of this imaging probe and its topography. According to $\mathrm{Li}$ et al., CQDs have been observed in different organs of mice after $6 \mathrm{~h}$ of their injection and showed bright fluorescence. Some of them are also observed in the brain, which indicates that CQDs can enter the brain and have the ability to cross the blood-brain barrier (Li N. et al., 2012). For the in vivo fluorescence imaging, polymer-coated nitrogen-doped CQDs can also be used, and these CQDs have the ability to enter the glioma cells. Because of the presence of hydrophilic polymer coating on the nitrogendoped CQD surface, they have enhanced ability to circulate in the bloodstream and target the site of interest of the cancerous area. This suggests that polymer-coated N-doped CQDs can enhance the blood-brain barrier crossing and then increase their accumulation for glioma imaging (Wang et al., 2015).

\section{Gene Delivery}

Gene therapy has considerable importance in biotechnology and medical sciences applications, based on the treatment of diseases and correction of expression of genes on DNA, encoded for missing or defective genes. Therefore, different types of nanoparticles are used in gene therapy to deliver genes at the targeted sites of DNA. In one study, theranostic and reducible nanoagents fc-rPEI-CQDs were developed and characterized. These nanoagents are highly biocompatible and are excellent gene delivery carriers for the treatment of targeted lung cancer by encapsulating siRNAs and releasing them in a reductive environment ( $\mathrm{Wu}$ et al., 2016). Cationic dual-function CQDs produced by the one-step microwave pyrolysis method have been used as self-imaging and non-viral gene delivery agents in chondrogenesis from fibroblasts. These cationic CQDs have favorable properties such as tunable fluorescence, less cytotoxicity, biocompatibility, and high quantum yield. Cationic CQDs can condense the gene vector SOX9 to form a very small nanostructure having a size of $10-30 \mathrm{~nm}$ for their application (Cao et al., 2018).

CQDs can be used as a gene carrier. According to a study, PCD and DNA were mixed to form the PCD/DNA complex. By agarose gel electrophoresis, the ability of CQDs to condense DNA was observed. Enhanced green fluorescent protein (EGFP) was used as a gene to visualize the in vivo gene expression. Emission of green fluorescence proved the expression and delivery of genes into the targeted site. PCD emitting blue fluorescence indicates that PCD could be used as a label for gene delivery and bioimaging because of this bright blue fluorescence and high transfection ability (Hu et al., 2014). In another study, polyethylenimine-functionalized CQDs (CQDsPEI) showed multicolor fluorescence having the ability of both gene delivery and bioimaging. CQD-PEI/DNA complexes were used for the delivery and labeling of COS-7 cells. After transfection, COS-7 cells had strong fluorescence emitted from CQDs-PEI dispersed in the cytosol. Passivated CQDs have the ability to emit different colors such as blue, green, and red at different excitation wavelengths and may have the potential to be used in in vivo fluorescence tracking (Liu et al., 2012).

\section{Photodynamic Therapy/Photothermal Therapy}

Photodynamic therapy has a great role in the treatment of cancers and many non-neoplastic lesions having less side effects and non-invasiveness. Implementation of PDT depends upon the photothermal coupling agents (Markovic et al., 2012). A very novel therapeutic agent DOX loaded, sgc8c aptamer conjugated, and SWCNTs-PEG-Fe3O4@CQDs with multifunction ability can target and kill cancer cells by releasing the drug photodynamically or photothermally. SWCNTs-PEG-Fe3O4@CQDs can convert $808 \mathrm{~nm}$ NIR into heat energy, generate ROS (reactive oxygen species), and remove cancer cells. They are biocompatible, hydrophilic, and highly stable as well as having excellent magnetic properties which make them suitable for magnetic resonance imaging and drug delivery. Thus, SWCNT-PEG-Fe3O4@CQD nano-carriers are favorable for the treatment of cervical cancer and other diseases that need precise drug targeting (Zhang et al., 2018).

For phototreatment, another CQD nano-composite has been reported for drug delivery to kill cancer cells. It is prepared hydrothermally, functionalized with folic acid (FA), and conjugated with a riboflavin (Rf) photosensitizer. Crosslinked chitosan complexes (Ch-Fe-CL) are used as precursors for their synthesis. Due to their less toxicity, CQD nano-composites (FeN@CQDs) are incorporated into polymer nano-spheres. Synthesized nano-composites (FeN@CQDs) are then loaded with chemotherapeutic molecules, DOX. Nano-composites loaded with chemotherapeutic molecules (DOX) show strong absorbance in the NIR region with higher efficiency of photothermal conversion, and NIR light can stimulate the release of the drug, allowing for accumulation of the drug into the cell and increasing the effectiveness to kill cancer cells (Zhang et al., 2017). CQDs and C60 fullerenes have been suggested as photosensitizer drugs. Photoexcited CQDs can get access to target cells easily by starting an autophagic response that helps to detect the photodynamic cytotoxicity (Markovic et al., 2012).

\section{Pharmaceutical Formulations}

Currently, CQDs are being used in many biological applications to replace toxic nanoparticles, but still there is not enough research on the impact of CQDs on peptides and protein fibrillation. In one study, CQDs have been prepared from carbon powder having a diameter of about $4 \mathrm{~nm}$. It was observed in experiments that CQDs have the ability to prevent the fibrillation of insulin depending upon the concentration of CQDs. According to this study, $40 \mu \mathrm{g}$ of CQDs per $\mathrm{ml}$ can inhibit insulin from fibrillation under $65^{\circ} \mathrm{C}$ for 5 days, but in the absence of CQDs, human insulin starts denaturing within $3 \mathrm{~h}$ in the same conditions. The fibrillation-preventing effect is because of the reaction between CQDs and insulin components including monomers and oligomers. Because of this inhibiting capability, CQDs are the potential candidate to be used in biological applications and in pharmaceutical industries for formulation of insulin (Li et al., 2015). 
In another study, three gel formulations loaded with N-hydroxyphthalimide CQDs (NHF-CDs) were developed to check their anticancer activities. Loaded gels were analyzed by rheological analysis. The results of the study indicated that NHFCD-loaded gels have more potential to affect the size, organization of cells, and number of spheroids as well as having a significant effect on multiplication and aggregation of tumors cells in spheroids (Savin et al., 2019).

\section{Antibacterial Effect for Treating Infection/ Inflammation}

Carbon quantum dot polyamines (CQDPAs) as an antibacterial agent have been synthesized from polyamines for the treatment of bacterial keratitis. These small-sized and highly cationic CQDs have strong antibacterial activity and can disrupt the bacterial membrane. They are a promising candidate for clinical applications in eye infection treatments and even for bacteriainduced infections (Jian et al., 2017). Quaternary ammonium CQDs have good antimicrobial activity against gram-positive bacteria and are synthesized by the green method using 2,3epoxypropyltrimethylammonium chloride and diallyldimethylammonium chloride. In mice, they were used to treat pneumonia, and their in vivo and in vitro toxicity test demonstrated that they are encouraging agents in the treatment of infection of gram-positive bacteria (Zhao et al., 2020).

Some bacteria have resistance against certain drugs, becoming very dangerous to human health and life. There is a need to synthesize antibacterial agents against these hazardous bacteria. One of them is N-doped CQDs, synthesized by the one-step method having antibacterial activity against Staphylococcus and MRSA with the ability to destroy their cell structures, but they do not have effectiveness against E. coli. Their mechanism of action demonstrates that positively charged N-doped CQDs interact with negatively charged bacteria and bind with Staphylococcus bacteria at specific sites. These N-doped CQDs used in wound infection caused by MRSA demonstrated the same effects as vancomycin (Zhao et al., 2020). CQDs synthesized from curcumin showed antiviral activity against enterovirus, and their activity depends upon heating temperature during synthesis. The results of this study showed that they are highly biocompatible and have strong inhibitory activity against viral infections in RD cells (Lin et al., 2019).

\section{DISCUSSION}

Since the discovery of CQDs, many facile, cheap, and efficient synthetic routes have been developed to produce photostable, biocompatible, non-blinking, photobleaching-resistant, and fluorescent CQDs. So far, there is mounting evidence that CQDs produce fluorescence by two mechanisms: one is associated with the bandgap transition of $\mathrm{sp}^{2}$ conjugated carbon and the other is the fluorescence from the surface defects (Cao et al., 2013; Molaei, 2019). The bandgap is created in a graphene sheet by forming $\mathrm{sp}^{2}$ domains in such a way that conjugated $\pi$ domains are isolated. The smaller the size of CQDs, the larger the bandgap emitting high energy (Cong and Zhao, 2018). They show strong absorption in the UV region but weak emissions. The second is obtained with surface defects which give out strong fluorescence due to surface energy trap formation. So, combined $\mathrm{sp}^{2}$ and $\mathrm{sp}^{3}$ hybridized carbon and functionalized surface defects help in producing multicolor emission. The fluorescence emission of CQDs can be tuned depending on the synthetic route taken for the preparation of CQDs (Adams and Fagbenro-Owoseni, 2017; Molaei, 2020). This helps in producing the required emission wavelength by knowing the absorption wavelength. Therefore, it can be applied in biolabeling and bioimaging as they are more photoluminescent as compared to conventional QDs (Wu et al., 2016). However, experimental findings are of little use as contradictory outcomes are commonly found. CQDs prepared from the hydrothermal method using citric acid and ethylenediamine produced $80 \%$ of QY (Zhu et al., 2013). The surface state of CQDs played a vital role in displaying wavelength-dependent photoluminescence (PL). In another study, CQDs prepared from the laser ablation method produced PL only after surface passivation showing a broad spectrum emission from visible to near IR (Sun et al., 2006). The effect of surface passivation on QY has also been studied. CQDs produced from carbohydrates by chemical oxidation showed weak PL but got stronger when TTDDA was used (Peng and Travas-Sejdic, 2009). CQD PL emission is also dependent on its pH value (Liu et al., 2007; Lakowicz, 2013). On the contrary, activated charcoal was used to prepare CQDs through chemical oxidation having QY $>10 \%$ that demonstrated strong electrochemiluminescence (ECL) up to $600 \mathrm{~nm}$ without any surface passivation (Dong et al., 2010). The results indicated that ECL is reduced with surface passivation and it is independent of the size as in other CQDs. To compare PL and ECL, CQDs were excited at $330 \mathrm{~nm}$ which emitted PL at $455 \mathrm{~nm}$ and ECL at $535 \mathrm{~nm}$ (Zheng et al., 2009). If CQDs were completely passivated, ECL would have the same profile as PL. UCPL is applicable mostly in bioimaging in the near-IR region because it can penetrate through tissue easily and emit fluorescence in the background (Cao et al., 2007).

Normally, surface passivation is done with a polymer, e.g., PEG1500N, after CQDs have been treated with an acid, but their surfaces are prone to oxidative erosion when strong acids are used (Sun et al., 2006). An experiment was conducted where CQDs prepared from hydrothermal reaction were reduced with $\mathrm{NaBH} 4$ producing 40.5\% QY (Shen et al., 2012). These non-passivated CQDs with high QY are a good alternative to acid treatment, but they are not exceedingly efficient and reproducible. Hence, acidtreated CQDs and surface passivation are more preferred to obtain maximum QY. Functionalization is another technique in achieving surface defects by adding functional groups to CQD surfaces resulting in variable fluorescence emission (Wang et al., 2012). CQDs functionalized with the PEI polymer have outstanding fluorescence properties and excitation-dependent emission characteristics that make them useful for multicolor bioimaging. CQDs obtained at high temperature are better than low-temperature CQDs because of their high QY and low toxicity in cells even at high concentrations (Kundu et al., 2018). 
Moreover, doping with heteroatoms has also helped in increasing the fluorescence of CQDs. 83\% of QY was reported with $\mathrm{N}$ and Mg doping (Li F. et al., 2014). Doping and surface passivation can be adopted efficiently to attain high QY. However, this brings contamination and environmental pollution because of the use of thin polymer coating on CQDs for surface passivation; therefore, it is not widely accepted. Along with QY, the photostability of CQDs is a very important aspect to consider. CQDs prepared from electrooxidation of graphite showed no change in fluorescence when exposed for $6 \mathrm{~h}$ in the Xe lamp $(8.3 \mathrm{~W})$ (Zhao et al., 2008).

Due to high biocompatibility and environmentally friendly conformation, CQDs would innately fit in the research area of bioimaging and biosensing such as electrochemical biosensing and detection of toxic chemicals. In order to compete with readily available fluorophores and semiconductor QDs, CQDs are required to have relatively high $\mathrm{QY}$ for their applications. Therefore, there are numerous synthetic routes available with high reproducibility and scalability with uniform structures and sizes to date. A large variety of studies have been conducted with both top-down and bottom-up methods depending on what is required of CQDs for their effective application. CQDs prepared by the laser ablation method using argon gas showed no fluorescence. Only after treatment with acid followed by functionalization with PEG and NAC, fluorescence emission was observed (Gonçalves et al., 2010). These were presented as biosensors for the detection of $\mathrm{Hg}(\mathrm{II})$. CQDs prepared by microwave pyrolysis using glycerol with PEI passivation have the ability to deliver genes because of the positive charge of the polymer layer on the surface of CQDs, and encapsulated CQDs emit fluorescence when used as a fluorescence probe in bioimaging (Liu et al., 2012). CQDs are being used as electrochemical biosensors in the detection of $\mathrm{H}_{2} \mathrm{O}_{2}, \mathrm{O}_{2}$, and a huge number of macromolecules with increased sensitivity and selectivity because of their excellent electrocatalytic properties, chemical stability, hydrophilic nature, and solubility in organic solvents (Kour et al., 2020). These excellent properties of CQDs also enable them to be used in optical sensing applications such as the detection of important analytes in the human fluid (Lu et al., 2016). With advancement in technology, simpler and green synthetic routes have been introduced. To reduce the cost of materials and increase the scalability of the product, CQDs produced from the one-step electrochemical method using graphite showed both up- and down-conversion luminescence (Ahirwar et al., 2017). In another study, CQDs produced from ethanol in the one-step electrochemical method showed desired fluorescence, solubility, and photostability (Li et al., 2011b). Subsequently, many cheap and biocompatible starting materials such as citrate (Guo et al., 2013), ascorbic acid (Sugiarti and Darmawan, 2015), orange juice (Sahu et al., 2012), chitosan (Yang et al., 2012), and gelatin (Jing, 2018) have been used in simple steps. The synthetic methods such as microwave pyrolysis or hydrothermal methods are highly appreciated to formulate size-controlled CQDs and avoid carbonaceous aggregation. Unique properties of CQDs obtained from food wastes are responsible for their usage in food analysis as well as in the detection of heavy metals, food chemicals, pathogens in food, and food additives (Fan et al., 2020; Carneiro et al., 2021).

CQDs have significantly altered the field of nano-medicine. So far, researchers have made extensive efforts to use CQDs to deliver the drug to the tumor-specific site using targeted receptors. They are also being used in pharmaceutical formulations to inhibit fibrillations of insulin and have significant potential to be used in biological systems and pharmaceutical industries for formulations and processing ( $\mathrm{Li}$ et al., 2015). Gel formulations have cell modulatory activities for cancer control in potential clinical applications by not affecting normal cells as they influence cancerous cells (Savin et al., 2019). Due to low toxicity, CQDs are seeking their place among innovative cancer treatment materials for theranostic applications ( $\mathrm{Li}$ S. et al., 2020). CQDs have a great role in photothermal and photodynamic therapy for targeted drug release in significantly advanced cancer treatment. Chemophototherapy which is a combination of PTT, PDT, and CT has the ability to increase the anticancer activity via synergistic effects (Zhang et al., 2018). The biodistribution and pharmacokinetics of CQDs have been studied thoroughly under various processes such as conjugation, passivation, and doping as these nano-composites have shown promising results in vitro as well as in vivo. Thus, their utility in therapeutics and biomedicine such as bioimaging, biosensing, diagnosis, gene delivery, and drug delivery is explored extensively. CQD-based hybrids and nanomaterials have abundantly been used in electrocatalytic sectors, electro-reduction, and electro-biosensing, and their wider production of a green, cost-effective electrode material can be advantageous in the future for many applications (Dave and Gomes, 2019). CQD hybrids have antibacterial and antiviral activity which provides a new direction in the field of medical sciences. They have been used in the treatment of cancers, tumors, inflammations, and many bacterial infections according to some reports, and it was observed that their inhibiting activity is way better than that of many antibiotics (Jian et al., 2017; Zhao et al., 2019).

\section{CONCLUSION AND FUTURE PROSPECTS}

Due to their properties and applications in various environmental and health-related fields, CQDs have garnered a lot of interest in the past few decades, however; there are still some problems that need to be addressed for their practical bioapplications such as preparation of CQDs, their sizes, reproducibility, and low quantum yield. Modifying the shapes, sizes, reproducibility, and specificity of the CQDs gives potential work for the future. The size of CQDs is responsible for their fluorescence properties, and also their low toxicity makes them able to be used in many in vivo applications. Moreover, preparation of CQD nanocomposites with surface functionalization and bandgap engineering expand their potential applications in various fields by overcoming their low quantum yield problem. It has been reported in the literature that CQDs can absorb light and have the ability to convert it into heat energy as well as 
generating reactive oxygen species, which allows them to be used as therapeutic agents as well as diagnostic agents without any use of complex theranostic systems. As it is very difficult to control the size of CQDs, there should be more research on the fabrication techniques that provide improved distribution of sizes, required morphology, and defect-free surfaces of CQDs for the desired biological activities as well as on the evaluation and comparison of CQDs fabricated by different methods using different sources. Regardless of the problems, CQDs with versatile nature, surface modifications, and outstanding properties have increased the interest of scientists to continue to explore them for their modifications and new hybrids of CQDs for the development of innovative applications in the field of biomedical sciences. Synthesis of CQDs has evolved from complex methods using conventional organic materials to convenient green synthesis. In addition, many research studies have been done to improve CQD functionality, but it is slightly difficult to use them as chemosensors and biosensors while increasing sensitivity and maintaining their selectivity. For their improved sensitivity and selectivity, suitable carbon precursors should be used that define physical and chemical properties of CQDs. CQDs are presently being used in multiple bioapplications including nano-medicine, bioimaging, biosensing, and biomarker because of their unique properties such as biocompatibility, low toxicity, chemical stability, hydrophilic nature, and most importantly tunable photoluminescence property. But according to our knowledge, there is very less research till now where CQDs are being used as labels or tags, but they are being used as carriers to labels and tags and are good alternative to semiconductor quantum dots because of their low toxicity and surface passivation. The semiconductor quantum dots have been replaced due to their biocompatibility and adjustable photoluminescence characteristics. It has been observed from

\section{REFERENCES}

Adams, L., and Fagbenro-Owoseni, K. (2017). Tunable Carbon Quantum Dots from Starch via Microwave Assisted Carbonization.

Ahirwar, S., Mallick, S., and Bahadur, D. (2017). Electrochemical Method to Prepare Graphene Quantum Dots and Graphene Oxide Quantum Dots. ACS omega 2 (11), 8343-8353. doi:10.1021/acsomega.7b01539

Anwar, S., Ding, H., Xu, M., Hu, X., Li, Z., Wang, J., et al. (2019). Recent Advances in Synthesis, Optical Properties, and Biomedical Applications of Carbon Dots. ACS Appl. Bio Mater. 2 (6), 2317-2338. doi:10.1021/acsabm.9b00112

Baker, S. N., and Baker, G. A. (2010). Luminescent Carbon Nanodots: Emergent Nanolights. Angew. Chem. Int. Edition 49 (38), 6726-6744. doi:10.1002/ anie. 200906623

Bao, L., Zhang, Z.-L., Tian, Z.-Q., Zhang, L., Liu, C., Lin, Y., et al. (2011). Electrochemical Tuning of Luminescent Carbon Nanodots: from Preparation to Luminescence Mechanism. Adv. Mater. 23 (48), 5801-5806. doi:10.1002/adma.201102866

Barati, A., Shamsipur, M., and Abdollahi, H. (2015). Hemoglobin Detection Using Carbon Dots as a Fluorescence Probe. Biosens. Bioelectron. 71, 470-475. doi:10.1016/j.bios.2015.04.073

Bhattacharya, S., Sarkar, R., Nandi, S., Porgador, A., and Jelinek, R. (2017). Detection of Reactive Oxygen Species by a Carbon-Dot-Ascorbic Acid Hydrogel. Anal. Chem. 89 (1), 830-836. doi:10.1021/ acs.analchem.6b03749 studies mentioned above that CQDs can lead to many innovations in the field of biomedical applications. One of these is to verify vaccination status by synthesizing long-term photostable, fluorescent, and non-photobleaching CQDs that can remain in the skin. Maintaining vaccination records is difficult, especially in developing regions, due to the lack of infrastructure. A dissolvable microneedle patch could be used to incorporate these CQDs into the skin, and through near-IR imaging, the record can be detected. The same approach may be used for people with allergies. In the event of an emergency, for example, when a patient is fainting or in an accident, physicians need to treat the patient as soon as possible. Testing for allergic responses can sometimes be the deciding factor between life and death before any medication like penicillin is administered. Therefore, having these CQD tags on hands will help them identify if the patient is allergic to anything. Cheap near-IR scanners can be developed on smartphones' cameras for this purpose. Similarly, next-generation radiology-imaging films and sensors can be synthesized, incorporating CQDs as well as radiation meters to have a qualitative account of radioactive contamination.

\section{AUTHOR CONTRIBUTIONS}

NA reviewed the manuscript, analyzed and edited the data. MN critically reviewed the manuscript. TJ compiled the data and prepared the first draft.

\section{ACKNOWLEDGMENTS}

No individuals other than the authors contributed in the manuscript.

Campuzano, S., Yáñez-Sedeño, P., and Pingarrón, J. M. (2019). Carbon Dots and Graphene Quantum Dots in Electrochemical Biosensing. Nanomaterials 9 (4), 634. doi:10.3390/nano9040634

Cao, L., Meziani, M. J., Sahu, S., and Sun, Y.-P. (2013). Photoluminescence Properties of Graphene versus Other Carbon Nanomaterials. Acc. Chem. Res. 46 (1), 171-180. doi:10.1021/ar300128j

Cao, L., Wang, X., Meziani, M. J., Lu, F., Wang, H., Luo, P. G., et al. (2007). Carbon Dots for Multiphoton Bioimaging. J. Am. Chem. Soc. 129 (37), 11318-11319. doi:10.1021/ja0735271

Cao, X., Wang, J., Deng, W., Chen, J., Wang, Y., Zhou, J., et al. (2018). Photoluminescent Cationic Carbon Dots as Efficient Non-viral Delivery of Plasmid SOX9 and Chondrogenesis of Fibroblasts. Scientific Rep. 8 (1), 1-11. doi:10.1038/s41598-018-25330-x

Carneiro, S. V., Holanda, M. H. B., Cunha, H. O., Oliveira, J. J. P., Pontes, S. M. A., Cruz, A. A. C., et al. (2021). Highly Sensitive Sensing of Food Additives Based on Fluorescent Carbon Quantum Dots. J. Photochem. Photobiol. A: Chem. 411, 113198. doi:10.1016/j.jphotochem.2021.113198

Chatzimitakos, T., Kasouni, A., Sygellou, L., Avgeropoulos, A., Troganis, A., and Stalikas, C. (2017). Two of a Kind but Different: Luminescent Carbon Quantum Dots from Citrus Peels for Iron and Tartrazine Sensing and Cell Imaging. Talanta 175, 305-312. doi:10.1016/j.talanta.2017.07.053

Chen, D., Zhuang, X., Zhai, J., Zheng, Y., Lu, H., and Chen, L. (2018). Preparation of Highly Sensitive Pt Nanoparticles-Carbon Quantum Dots/ionic Liquid Functionalized Graphene Oxide Nanocomposites and Application for H2O2 Detection. Sensors Actuators B: Chem. 255, 1500-1506. doi:10.1016/j.snb.2017.08.156 
Choi, Y., Thongsai, N., Chae, A., Jo, S., Kang, E. B., Paoprasert, P., et al. (2017). Microwave-assisted Synthesis of Luminescent and Biocompatible Lysine-Based Carbon Quantum Dots. J. Ind. Eng. Chem. 47, 329-335. doi:10.1016/ j.jiec.2016.12.002

Cong, S., and Zhao, Z. (2018). Carbon Quantum Dots: A Component of Efficient Visible Light Photocatalysts. London, UK: InTech. doi:10.5772/ intechopen.70801

Costas-Mora, I., Romero, V., Lavilla, I., and Bendicho, C. (2014). In Situ building of a Nanoprobe Based on Fluorescent Carbon Dots for Methylmercury Detection. Anal. Chem. 86 (9), 4536-4543. doi:10.1021/ac500517h

Dang, H., Huang, L.-K., Zhang, Y., Wang, C.-F., and Chen, S. (2016). Large-scale Ultrasonic Fabrication of white Fluorescent Carbon Dots. Ind. Eng. Chem. Res. 55 (18), 5335-5341. doi:10.1021/acs.iecr.6b00894

Dave, K., and Gomes, V. G. (2019). Carbon Quantum Dot-Based Composites for Energy Storage and Electrocatalysis: Mechanism, Applications and Future Prospects. Nano Energy 66, 104093.

Deng, J., Lu, Q., Mi, N., Li, H., Liu, M., Xu, M., et al. (2014). Electrochemical Synthesis of Carbon Nanodots Directly from Alcohols. Chem. Eur. J. 20 (17), 4993-4999. doi:10.1002/chem.201304869

Dimos, K. (2016). Carbon Quantum Dots: Surface Passivation and Functionalization. Coc 20 (6), 682-695. doi:10.2174/1385272819666150730220948

Doñate-Buendia, C., Torres-Mendieta, R., Pyatenko, A., Falomir, E., FernándezAlonso, M., and Mínguez-Vega, G. (2018). Fabrication by Laser Irradiation in a Continuous Flow Jet of Carbon Quantum Dots for Fluorescence Imaging. ACS omega 3 (3), 2735-2742. doi:10.1021/acsomega.7b02082

Dong, Y., Zhou, N., Lin, X., Lin, J., Chi, Y., and Chen, G. (2010). Extraction of Electrochemiluminescent Oxidized Carbon Quantum Dots from Activated Carbon. Chem. Mater. 22 (21), 5895-5899. doi:10.1021/cm1018844

Emam, A. N., Loutfy, S. A., Mostafa, A. A., Awad, H., and Mohamed, M. B. (2017). Cyto-toxicity, Biocompatibility and Cellular Response of Carbon DotsPlasmonic Based Nano-Hybrids for Bioimaging. RSC Adv. 7 (38), 23502-23514. doi:10.1039/c7ra01423f

Fan, H., Zhang, M., Bhandari, B., and Yang, C.-h. (2020). Food Waste as a Carbon Source in Carbon Quantum Dots Technology and Their Applications in Food Safety Detection. Trends Food Sci. Tech. 95, 86-96. doi:10.1016/ j.tifs.2019.11.008

Gao, L., Zhao, X., Wang, J., Wang, Y., Yu, L., Peng, H., et al. (2018). Multiple Functionalized Carbon Quantum Dots for Targeting Glioma and Tissue Imaging. Opt. Mater. 75, 764-769. doi:10.1016/j.optmat.2017.11.044

Gonçalves, H., Jorge, P. A. S., Fernandes, J. R. A., and Esteves da Silva, J. C. G. (2010). Hg(II) Sensing Based on Functionalized Carbon Dots Obtained by Direct Laser Ablation. Sensors Actuators B: Chem. 145 (2), 702-707. doi:10.1016/j.snb.2010.01.031

Gude, V. (2014). Synthesis of Hydrophobic Photoluminescent Carbon Nanodots by Using L-Tyrosine and Citric Acid through a thermal Oxidation Route. Beilstein J. Nanotechnol. 5, 1513-1522. doi:10.3762/bjnano.5.164

Guo, Y., Wang, Z., Shao, H., and Jiang, X. (2013). Hydrothermal Synthesis of Highly Fluorescent Carbon Nanoparticles from Sodium Citrate and Their Use for the Detection of Mercury Ions. Carbon 52, 583-589. doi:10.1016/ j.carbon.2012.10.028

Holá, K., Sudolská, M., Kalytchuk, S., Nachtigallová, D., Rogach, A. L., Otyepka, M., et al. (2017). Graphitic Nitrogen Triggers Red Fluorescence in Carbon Dots. ACS nano 11 (12), 12402-12410. doi:10.1021/acsnano.7b06399

Hu, L., Sun, Y., Li, S., Wang, X., Hu, K., Wang, L., et al. (2014). Multifunctional Carbon Dots with High Quantum Yield for Imaging and Gene Delivery. Carbon 67, 508-513. doi:10.1016/j.carbon.2013.10.023

Hu, S.-L., Niu, K.-Y., Sun, J., Yang, J., Zhao, N.-Q., and Du, X.-W. (2009). One-step Synthesis of Fluorescent Carbon Nanoparticles by Laser Irradiation. J. Mater. Chem. 19 (4), 484-488. doi:10.1039/b812943f

Hu, S., Liu, J., Yang, J., Wang, Y., and Cao, S. (2011). Laser Synthesis and Size Tailor of Carbon Quantum Dots. J. Nanopart Res. 13 (12), 7247-7252. doi:10.1007/ s11051-011-0638-y

Hua, X.-W., Bao, Y.-W., Chen, Z., and Wu, F.-G. (2017). Carbon Quantum Dots with Intrinsic Mitochondrial Targeting Ability for Mitochondria-Based Theranostics. Nanoscale 9 (30), 10948-10960. doi:10.1039/c7nr03658b

Huang, C.-C., Hung, Y.-S., Weng, Y.-M., Chen, W., and Lai, Y.-S. (2019). Sustainable Development of Carbon Nanodots Technology: Natural
Products as a Carbon Source and Applications to Food Safety. Trends Food Sci. Tech. 86, 144-152. doi:10.1016/j.tifs.2019.02.016

Huang, C., Dong, H., Su, Y., Wu, Y., Narron, R., and Yong, Q. (2019). Synthesis of Carbon Quantum Dot Nanoparticles Derived from Byproducts in Bio-Refinery Process for Cell Imaging and In Vivo Bioimaging. Nanomaterials 9 (3), 387. doi:10.3390/nano9030387

Jia, X., Li, J., and Wang, E. (2012). One-pot green Synthesis of Optically pHSensitive Carbon Dots with Upconversion Luminescence. Nanoscale 4 (18), 5572-5575. doi:10.1039/c2nr31319g

Jia, X., Pei, M., Zhao, X., Tian, K., Zhou, T., and Liu, P. (2016). PEGylated Oxidized Alginate-DOX Prodrug Conjugate Nanoparticles Cross-Linked with Fluorescent Carbon Dots for Tumor Theranostics. ACS Biomater. Sci. Eng. 2 (9), 1641-1648. doi:10.1021/acsbiomaterials.6b00443

Jian, H.-J., Wu, R.-S., Lin, T.-Y., Li, Y.-J., Lin, H.-J., Harroun, S. G., et al. (2017). Super-cationic Carbon Quantum Dots Synthesized from Spermidine as an Eye Drop Formulation for Topical Treatment of Bacterial Keratitis. ACS nano 11 (7), 6703-6716. doi:10.1021/acsnano.7b01023

Jing, A. (2018). The Hydrothermal Synthesis of Quantum Dots Using Gelatin.

Kong, B., Zhu, A., Ding, C., Zhao, X., Li, B., and Tian, Y. (2012). Carbon Dot-Based Inorganic-Organic Nanosystem for Two-Photon Imaging and Biosensing of $\mathrm{pH}$ Variation in Living Cells and Tissues. Adv. Mater. 24 (43), 5844-5848. doi:10.1002/adma.201202599

Kour, R., Arya, S., Young, S.-J., Gupta, V., Bandhoria, P., and Khosla, A. (2020). Review-Recent Advances in Carbon Nanomaterials as Electrochemical Biosensors. J. Electrochem. Soc. 167 (3), 037555. doi:10.1149/1945-7111/ab6bc4

Kundu, A., Lee, J., Park, B., Ray, C., Sankar, K. V., Kim, W. S., et al. (2018). Facile Approach to Synthesize Highly Fluorescent Multicolor Emissive Carbon Dots via Surface Functionalization for Cellular Imaging. J. Colloid Interf. Sci. 513, 505-514. doi:10.1016/j.jcis.2017.10.095

Lakowicz, J. R. (2013). Principles of Fluorescence Spectroscopy. Springer science \& business media.

Lee, H. U., Park, S. Y., Park, E. S., Son, B., Lee, S. C., Lee, J. W., et al. (2014). Photoluminescent Carbon Nanotags from Harmful Cyanobacteria for Drug Delivery and Imaging in Cancer Cells. Scientific Rep. 4, 4665. doi:10.1038/ srep04665

Li, F., Liu, C., Yang, J., Wang, Z., Liu, W., and Tian, F. (2014a). Mg/N Double Doping Strategy to Fabricate Extremely High Luminescent Carbon Dots for Bioimaging. RSC Adv. 4 (7), 3201-3205. doi:10.1039/c3ra43826k

Li, H., He, X., Liu, Y., Huang, H., Lian, S., Lee, S.-T., et al. (2011a). One-step Ultrasonic Synthesis of Water-Soluble Carbon Nanoparticles with Excellent Photoluminescent Properties. Carbon 49 (2), 605-609. doi:10.1016/ j.carbon.2010.10.004

Li, H., Liu, R., Liu, Y., Huang, H., Yu, H., Ming, H., et al. (2012a). Carbon Quantum dots/Cu2O Composites with Protruding Nanostructures and Their Highly Efficient (Near) Infrared Photocatalytic Behavior. J. Mater. Chem. 22 (34), 17470-17475. doi:10.1039/c2jm32827e

Li, H., Ming, H., Liu, Y., Yu, H., He, X., Huang, H., et al. (2011b). Fluorescent Carbon Nanoparticles: Electrochemical Synthesis and Their $\mathrm{pH}$ Sensitive Photoluminescence Properties. New J. Chem. 35 (11), 2666-2670. doi:10.1039/clnj20575g

Li, H., Zhang, Y., Wang, L., Tian, J., and Sun, X. (2011c). Nucleic Acid Detection Using Carbon Nanoparticles as a Fluorescent Sensing Platform. Chem. Commun. 47 (3), 961-963. doi:10.1039/c0cc04326e

Li, L.-L., Ji, J., Fei, R., Wang, C.-Z., Lu, Q., Zhang, J.-R., et al. (2012b). A Facile Microwave Avenue to Electrochemiluminescent Two-Color Graphene Quantum Dots. Adv. Funct. Mater. 22 (14), 2971-2979. doi:10.1002/ adfm. 201200166

Li, N., Liang, X., Wang, L., Li, Z., Li, P., Zhu, Y., et al. (2012c). Biodistribution Study of Carbogenic Dots in Cells and In Vivo for Optical Imaging. J. Nanoparticle Res. 14 (10), 1177. doi:10.1007/s11051-012-1177-x

Li, S., Su, W., Wu, H., Yuan, T., Yuan, C., Liu, J., et al. (2020a). Targeted Tumour Theranostics in Mice via Carbon Quantum Dots Structurally Mimicking Large Amino Acids. Nat. Biomed. Eng. 4, 704-716. doi:10.1038/s41551-020$0540-y$

Li, S., Wang, L., Chusuei, C. C., Suarez, V. M., Blackwelder, P. L., Micic, M., et al. (2015). Nontoxic Carbon Dots Potently Inhibit Human Insulin Fibrillation. Chem. Mater. 27 (5), 1764-1771. doi:10.1021/cm504572b 
Li, W., Wei, Z., Wang, B., Liu, Y., Song, H., Tang, Z., et al. (2020b). Carbon Quantum Dots Enhanced the Activity for the Hydrogen Evolution Reaction in Ruthenium-Based Electrocatalysts. Mater. Chem. Front. 4 (1), 277-284. doi:10.1039/c9qm00618d

Li, X., Wang, H., Shimizu, Y., Pyatenko, A., Kawaguchi, K., and Koshizaki, N. (2010). Preparation of Carbon Quantum Dots with Tunable Photoluminescence by Rapid Laser Passivation in Ordinary Organic Solvents. Chem. Commun. (Camb) 47 (3), 932-934. doi:10.1039/ c0cc03552a

Li, X., Robinson, S. M., Gupta, A., Saha, K., Jiang, Z., Moyano, D. F., et al. (2014b). Functional Gold Nanoparticles as Potent Antimicrobial Agents against MultiDrug-Resistant Bacteria. ACS nano 8 (10), 10682-10686. doi:10.1021/ nn5042625

Lin, C. J., Chang, L., Chu, H. W., Lin, H. J., Chang, P. C., Wang, R. Y. L., et al. (2019). High Amplification of the Antiviral Activity of Curcumin through Transformation into Carbon Quantum Dots. Small 15 (41), 1902641. doi:10.1002/smll.201902641

Lin, L., and Zhang, S. (2012). Creating High Yield Water Soluble Luminescent Graphene Quantum Dots via Exfoliating and Disintegrating Carbon Nanotubes and Graphite Flakes. Chem. Commun. 48 (82), 10177-10179. doi:10.1039/ c2cc35559k

Liu, C., Tang, B., Zhang, S., Zhou, M., Yang, M., Liu, Y., et al. (2018). Photoinduced Electron Transfer Mediated by Coordination between Carboxyl on Carbon Nanodots and Cu2+ Quenching Photoluminescence. J. Phys. Chem. C 122 (6), 3662-3668. doi:10.1021/acs.jpcc.7b12681

Liu, C., Zhang, P., Zhai, X., Tian, F., Li, W., Yang, J., et al. (2012). Nano-carrier for Gene Delivery and Bioimaging Based on Carbon Dots with PEI-Passivation Enhanced Fluorescence. Biomaterials 33 (13), 3604-3613. doi:10.1016/ j.biomaterials.2012.01.052

Liu, H., Ye, T., and Mao, C. (2007). Fluorescent Carbon Nanoparticles Derived from Candle Soot. Angew. Chem. 119 (34), 6593-6595. doi:10.1002/ ange.200701271

Liu, Q., Guo, B., Rao, Z., Zhang, B., and Gong, J. R. (2013). Strong Two-PhotonInduced Fluorescence from Photostable, Biocompatible Nitrogen-Doped Graphene Quantum Dots for Cellular and Deep-Tissue Imaging. Nano Lett. 13 (6), 2436-2441. doi:10.1021/nl400368v

Liu, X., Zhang, N., Bing, T., and Shangguan, D. (2014a). Carbon Dots Based DualEmission Silica Nanoparticles as a Ratiometric Nanosensor for Cu2+. Anal. Chem. 86 (5), 2289-2296. doi:10.1021/ac404236y

Liu, X., Zhang, N., Bing, T., and Shangguan, D. (2014b). Carbon Dots Based DualEmission Silica Nanoparticles as a Ratiometric Nanosensor for Cu2+. Anal. Chem. 86 (5), 2289-2296. doi:10.1021/ac404236y

Lu, S., Li, G., Lv, Z., Qiu, N., Kong, W., Gong, P., et al. (2016). Facile and Ultrasensitive Fluorescence Sensor Platform for Tumor Invasive Biomaker $\beta$-glucuronidase Detection and Inhibitor Evaluation with Carbon Quantum Dots Based on Inner-Filter Effect. Biosens. Bioelectron. 85, 358-362. doi:10.1016/j.bios.2016.05.021

Lu, S., Sui, L., Wu, M., Zhu, S., Yong, X., and Yang, B. (2019). Graphitic Nitrogen and High-Crystalline Triggered Strong Photoluminescence and RoomTemperature Ferromagnetism in Carbonized Polymer Dots. Adv. Sci. 6 (2), 1801192. doi:10.1002/advs.201801192

Markovic, Z. M., Ristic, B. Z., Arsikin, K. M., Klisic, D. G., Harhaji-Trajkovic, L. M., Todorovic-Markovic, B. M., et al. (2012). Graphene Quantum Dots as Autophagy-Inducing Photodynamic Agents. Biomaterials 33 (29), 7084-7092. doi:10.1016/j.biomaterials.2012.06.060

Mehta, V. N., Jha, S., Basu, H., Singhal, R. K., and Kailasa, S. K. (2015). One-step Hydrothermal Approach to Fabricate Carbon Dots from Apple Juice for Imaging of mycobacterium and Fungal Cells. Sensors Actuators B: Chem. 213, 434-443. doi:10.1016/j.snb.2015.02.104

Molaei, M. J. (2019). A Review on Nanostructured Carbon Quantum Dots and Their Applications in Biotechnology, Sensors, and Chemiluminescence. Talanta 196, 456-478. doi:10.1016/j.talanta.2018.12.042

Molaei, M. J. (2020). Principles, Mechanisms, and Application of Carbon Quantum Dots in Sensors: a Review. Anal. Methods 12 (10), 1266-1287. doi:10.1039/ c9ay02696g

Nguyen, V., Zhao, N., Yan, L., Zhong, P., Nguyen, V. C., and Le, P. H. (2020). Double-pulse Femtosecond Laser Ablation for Synthesis of Ultrasmall Carbon Nanodots. Mater. Res. Express 7 (1), 015606. doi:10.1088/2053-1591/ab6124
Niu, F., Ying, Y.-L., Hua, X., Niu, Y., Xu, Y., and Long, Y.-T. (2018). Electrochemically Generated green-fluorescent N-Doped Carbon Quantum Dots for Facile Monitoring Alkaline Phosphatase Activity Based on the Fe3+-Mediating ON-OFF-ON-OFF Fluorescence Principle. Carbon 127, 340-348. doi:10.1016/j.carbon.2017.10.097

Pan, L., Sun, S., Zhang, A., Jiang, K., Zhang, L., Dong, C., et al. (2015). Truly Fluorescent Excitation-dependent Carbon Dots and Their Applications in Multicolor Cellular Imaging and Multidimensional Sensing. Adv. Mater. 27 (47), 7782-7787. doi:10.1002/adma.201503821

Pan, M., Xie, X., Liu, K., Yang, J., Hong, L., and Wang, S. (2020). Fluorescent Carbon Quantum Dots-Synthesis, Functionalization and Sensing Application in Food Analysis. Nanomaterials 10 (5), 930. doi:10.3390/nano10050930

Park, S. Y., Lee, H. U., Park, E. S., Lee, S. C., Lee, J.-W., Jeong, S. W., et al. (2014). Photoluminescent green Carbon Nanodots from Food-Waste-Derived Sources: Large-Scale Synthesis, Properties, and Biomedical Applications. ACS Appl. Mater. Inter. 6 (5), 3365-3370. doi:10.1021/am500159p

Peng, H., and Travas-Sejdic, J. (2009). Simple Aqueous Solution Route to Luminescent Carbogenic Dots from Carbohydrates. Chem. Mater. 21 (23), 5563-5565. doi:10.1021/cm901593y

Peng, Z., Han, X., Li, S., Al-Youbi, A. O., Bashammakh, A. S., El-Shahawi, M. S., et al. (2017). Carbon Dots: Biomacromolecule Interaction, Bioimaging and Nanomedicine. Coord. Chem. Rev. 343, 256-277. doi:10.1016/j.ccr.2017.06.001

Qi, H., Teng, M., Liu, M., Liu, S., Li, J., Yu, H., et al. (2019). Biomass-derived Nitrogen-Doped Carbon Quantum Dots: Highly Selective Fluorescent Probe for Detecting Fe3+ Ions and Tetracyclines. J. Colloid Interf. Sci. 539, 332-341. doi:10.1016/j.jcis.2018.12.047

Qu, D., Zheng, M., Du, P., Zhou, Y., Zhang, L., Li, D., et al. (2013). Highly Luminescent S, N Co-doped Graphene Quantum Dots with Broad Visible Absorption Bands for Visible Light Photocatalysts. Nanoscale 5 (24), 12272-12277. doi:10.1039/c3nr04402e

Ray, S. C., Saha, A., Jana, N. R., and Sarkar, R. (2009). Fluorescent Carbon Nanoparticles: Synthesis, Characterization, and Bioimaging Application. J. Phys. Chem. C 113 (43), 18546-18551. doi:10.1021/jp905912n

Rosenholm, J. M., Sahlgren, C., and Lindén, M. (2010). Towards Multifunctional, Targeted Drug Delivery Systems Using Mesoporous Silica Nanoparticles Opportunities \& Challenges. Nanoscale 2 (10), 1870-1883. doi:10.1039/c0nr00156b

Sahu, S., Behera, B., Maiti, T. K., and Mohapatra, S. (2012). Simple One-step Synthesis of Highly Luminescent Carbon Dots from orange Juice: Application as Excellent Bio-Imaging Agents. Chem. Commun. 48 (70), 8835-8837. doi:10.1039/c2cc33796g

Sarkar, N., Sahoo, G., Das, R., Prusty, G., and Swain, S. K. (2017). Carbon Quantum Dot Tailored Calcium Alginate Hydrogel for $\mathrm{pH}$ Responsive Controlled Delivery of Vancomycin. Eur. J. Pharm. Sci. 109, 359-371. doi:10.1016/j.ejps.2017.08.015

Savin, C.-L., Tiron, C., Carasevici, E., Stan, C. S., Ibanescu, S. A., Simionescu, B. C., et al. (2019). Entrapment of N-Hydroxyphthalimide Carbon Dots in Different Topical Gel Formulations: New Composites with Anticancer Activity. Pharmaceutics 11 (7), 303. doi:10.3390/pharmaceutics11070303

Sharma, G., Anabousi, S., Ehrhardt, C., and Ravi Kumar, M. N. V. (2006). Liposomes as Targeted Drug Delivery Systems in the Treatment of Breast Cancer. J. Drug Target. 14 (5), 301-310. doi:10.1080/ 10611860600809112

Shen, J., Zhu, Y., Chen, C., Yang, X., and Li, C. (2011). Facile Preparation and Upconversion Luminescence of Graphene Quantum Dots. Chem. Commun. 47 (9), 2580-2582. doi:10.1039/c0cc04812g

Shen, L., Zhang, L., Chen, M., Chen, X., and Wang, J. (2013). The Production of $\mathrm{pH}$-Sensitive Photoluminescent Carbon Nanoparticles by the Carbonization of Polyethylenimine and Their Use for Bioimaging. Carbon 55, 343-349. doi:10.1016/j.carbon.2012.12.074

Shen, R., Song, K., Liu, H., Li, Y., and Liu, H. (2012). Dramatic Fluorescence Enhancement of Bare Carbon Dots through Facile Reduction Chemistry. ChemPhysChem 13 (15), 3549-3555. doi:10.1002/cphc.201200018

Shereema, R. M., Sankar, V., Raghu, K. G., Rao, T. P., and Shankar, S. S. (2015). One Step green Synthesis of Carbon Quantum Dots and its Application towards the Bioelectroanalytical and Biolabeling Studies. Electrochimica Acta 182, 588-595. doi:10.1016/j.electacta.2015.09.145

Shi, W., Wang, Q., Long, Y., Cheng, Z., Chen, S., Zheng, H., et al. (2011). Carbon Nanodots as Peroxidase Mimetics and Their Applications to Glucose Detection. Chem. Commun. 47 (23), 6695-6697. doi:10.1039/c1cc11943e 
Soroceanu, L., Gillespie, Y., Khazaeli, M. B., and Sontheimer, H. (1998). Use of Chlorotoxin for Targeting of Primary Brain Tumors. Cancer Res. 58 (21), 4871-4879.

Sugiarti, S., and Darmawan, N. (2015). Synthesis of Fluorescence Carbon Nanoparticles from Ascorbic Acid. Indones. J. Chem. 15 (2), 141-145. doi:10.22146/ijc.21207

Sun, Y.-P., Zhou, B., Lin, Y., Wang, W., Fernando, K. A. S., Pathak, P., et al. (2006). Quantum-sized Carbon Dots for Bright and Colorful Photoluminescence. J. Am. Chem. Soc. 128 (24), 7756-7757. doi:10.1021/ja062677d

Tian, L., Ghosh, D., Chen, W., Pradhan, S., Chang, X., and Chen, S. (2009). Nanosized Carbon Particles from Natural Gas Soot. Chem. Mater. 21 (13), 2803-2809. doi:10.1021/cm900709w

Titirici, M.-M., and Antonietti, M. (2010). Chemistry and Materials Options of Sustainable Carbon Materials Made by Hydrothermal Carbonization. Chem. Soc. Rev. 39 (1), 103-116. doi:10.1039/b819318p

Wang, D. M., Lin, K. L., and Huang, C. Z. (2019a). Carbon Dots-Involved Chemiluminescence: Recent Advances and Developments. Luminescence 34 (1), 4-22. doi:10.1002/bio.3570

Wang, F., Xie, Z., Zhang, H., Liu, C.-y., and Zhang, Y.-g. (2011a). Highly Luminescent Organosilane-Functionalized Carbon Dots. Adv. Funct. Mater. 21 (6), 1027-1031. doi:10.1002/adfm.201002279

Wang, J., Wang, C.-F., and Chen, S. (2012). Amphiphilic Egg-Derived Carbon Dots: Rapid Plasma Fabrication, Pyrolysis Process, and Multicolor Printing Patterns. Angew. Chem. Int. Ed. 51 (37), 9297-9301. doi:10.1002/ anie. 201204381

Wang, X., Cao, L., Lu, F., Meziani, M. J., Li, H., Qi, G., et al. (2009). Photoinduced Electron Transfers with Carbon Dots. Chem. Commun. 7, 3774-3776. doi:10.1039/b906252a

Wang, X., Cao, L., Yang, S.-T., Lu, F., Meziani, M. J., Tian, L., et al. (2010). Bandgap-Like Strong Fluorescence in Functionalized Carbon Nanoparticles. Angew. Chem. Int. Edition 49 (31), 5310-5314. doi:10.1002/anie.201000982

Wang, X., Feng, Y., Dong, P., and Huang, J. (2019b). A Mini Review on Carbon Quantum Dots: Preparation, Properties, and Electrocatalytic Application. Front. Chem. 7, 671. doi:10.3389/fchem.2019.00671

Wang, X., Yang, P., Feng, Q., Meng, T., Wei, J., Xu, C., et al. (2019c). Green Preparation of Fluorescent Carbon Quantum Dots from Cyanobacteria for Biological Imaging. Polymers 11 (4), 616. doi:10.3390/polym11040616

Wang, Y., Anilkumar, P., Cao, L., Liu, J.-H., Luo, P. G., Tackett, K. N., et al. (2011b). Carbon Dots of Different Composition and Surface Functionalization: Cytotoxicity Issues Relevant to Fluorescence Cell Imaging. Exp. Biol. Med. (Maywood) 236 (11), 1231-1238. doi:10.1258/ebm.2011.011132

Wang, Y., Bao, L., Liu, Z., and Pang, D.-W. (2011c). Aptamer Biosensor Based on Fluorescence Resonance Energy Transfer from Upconverting Phosphors to Carbon Nanoparticles for Thrombin Detection in Human Plasma. Anal. Chem. 83 (21), 8130-8137. doi:10.1021/ac201631b

Wang, Y., and Hu, A. (2014). Carbon Quantum Dots: Synthesis, Properties and Applications. J. Mater. Chem. C 2 (34), 6921. doi:10.1039/c4tc00988f

Wang, Y., Meng, Y., Wang, S., Li, C., Shi, W., Chen, J., et al. (2015). Direct SolventDerived Polymer-Coated Nitrogen-Doped Carbon Nanodots with High Water Solubility for Targeted Fluorescence Imaging of Glioma. Small 11 (29), 3575-3581. doi:10.1002/smll.201403718

Wei, W., Xu, C., Ren, J., Xu, B., and Qu, X. (2012). Sensing Metal Ions with Ion Selectivity of a crown Ether and Fluorescence Resonance Energy Transfer between Carbon Dots and Graphene. Chem. Commun. 48 (9), 1284-1286. doi:10.1039/c2cc16481g

Wen, X., Yu, P., Toh, Y.-R., Ma, X., and Tang, J. (2014). On the Upconversion Fluorescence in Carbon Nanodots and Graphene Quantum Dots. Chem. Commun. 50 (36), 4703-4706. doi:10.1039/c4cc01213e

Wu, X., Sun, S., Wang, Y., Zhu, J., Jiang, K., Leng, Y., et al. (2017). A Fluorescent Carbon-Dots-Based Mitochondria-Targetable Nanoprobe for Peroxynitrite Sensing in Living Cells. Biosens. Bioelectron. 90, 501-507. doi:10.1016/ j.bios.2016.10.060

Wu, Y.-F., Wu, H.-C., Kuan, C.-H., Lin, C.-J., Wang, L.-W., Chang, C.-W., et al. (2016). Multi-functionalized Carbon Dots as Theranostic Nanoagent for Gene Delivery in Lung Cancer Therapy. Scientific Rep. 6, 21170. doi:10.1038/ srep2 1170
Xu, G., Mahajan, S., Roy, I., and Yong, K.-T. (2013). Theranostic Quantum Dots for Crossing Blood-Brain Barrier In Vitro and Providing Therapy of HIVAssociated Encephalopathy. Front. Pharmacol. 4, 140. doi:10.3389/ fphar.2013.00140

Xu, X., Ray, R., Gu, Y., Ploehn, H. J., Gearheart, L., Raker, K., et al. (2004). Electrophoretic Analysis and Purification of Fluorescent Single-Walled Carbon Nanotube Fragments. J. Am. Chem. Soc. 126 (40), 12736-12737. doi:10.1021/ ja040082h

Xue, B., Yang, Y., Sun, Y., Fan, J., Li, X., and Zhang, Z. (2019a). Photoluminescent Lignin Hybridized Carbon Quantum Dots Composites for Bioimaging Applications. Int. J. Biol. Macromolecules 122, 954-961. doi:10.1016/ j.ijbiomac.2018.11.018

Xue, B., Yang, Y., Sun, Y., Fan, J., Li, X., and Zhang, Z. (2019b). Photoluminescent Lignin Hybridized Carbon Quantum Dots Composites for Bioimaging Applications. Int. J. Biol. macromolecules 122, 954-961. doi:10.1016/ j.ijbiomac.2018.11.018

Yang, S.-T., Cao, L., Luo, P. G., Lu, F., Wang, X., Wang, H., et al. (2009a). Carbon Dots for Optical Imaging In Vivo. J. Am. Chem. Soc. 131 (32), 11308-11309. doi:10.1021/ja904843x

Yang, S.-T., Wang, X., Wang, H., Lu, F., Luo, P. G., Cao, L., et al. (2009b). Carbon Dots as Nontoxic and High-Performance Fluorescence Imaging Agents. J. Phys. Chem. C 113 (42), 18110-18114. doi:10.1021/jp9085969

Yang, S.-T., Wang, X., Wang, H., Lu, F., Luo, P. G., Cao, L., et al. (2009). Carbon Dots as Nontoxic and High-Performance Fluorescence Imaging Agents. J. Phys. Chem. C 113 (42), 18110-18114. doi:10.1021/jp9085969

Yang, S., Sun, J., Li, X., Zhou, W., Wang, Z., He, P., et al. (2014). Large-scale Fabrication of Heavy Doped Carbon Quantum Dots with TunablePhotoluminescence and Sensitive Fluorescence Detection. J. Mater. Chem. A. 2 (23), 8660-8667. doi:10.1039/c4ta00860j

Yang, Y., Cui, J., Zheng, M., Hu, C., Tan, S., Xiao, Y., et al. (2012). One-step Synthesis of Amino-Functionalized Fluorescent Carbon Nanoparticles by Hydrothermal Carbonization of Chitosan. Chem. Commun. 48 (3), 380-382. doi:10.1039/c1cc15678k

Yang, Y., Yan, Z., Wei, D., Zhong, J., Liu, L., Zhang, L., et al. (2013). Tumorpenetrating Peptide Functionalization Enhances the Anti-glioblastoma Effect of Doxorubicin Liposomes. Nanotechnology 24 (40), 405101. doi:10.1088/09574484/24/40/405101

Zhai, X., Zhang, P., Liu, C., Bai, T., Li, W., Dai, L., et al. (2012). Highly Luminescent Carbon Nanodots by Microwave-Assisted Pyrolysis. Chem. Commun. 48 (64), 7955-7957. doi: $10.1039 /$ c2cc33869f

Zhang, M., Wang, W., Cui, Y., Chu, X., Sun, B., Zhou, N., et al. (2018). Magnetofluorescent Fe3O4/carbon Quantum Dots Coated Single-Walled Carbon Nanotubes as Dual-Modal Targeted Imaging and Chemo/ photodynamic/photothermal Triple-Modal Therapeutic Agents. Chem. Eng. J. 338, 526-538. doi:10.1016/j.cej.2018.01.081

Zhang, M., Wang, W., Zhou, N., Yuan, P., Su, Y., Shao, M., et al. (2017). Nearinfrared Light Triggered Photo-Therapy, in Combination with Chemotherapy Using Magnetofluorescent Carbon Quantum Dots for Effective Cancer Treating. Carbon 118, 752-764. doi:10.1016/ j.carbon.2017.03.085

Zhang, X., Zhang, Y., Wang, Y., Kalytchuk, S., Kershaw, S. V., Wang, Y., et al. (2013). Color-switchable Electroluminescence of Carbon Dot Light-Emitting Diodes. ACS nano 7 (12), 11234-11241. doi:10.1021/ nn $405017 \mathrm{q}$

Zhao, C., Wang, X., Wu, L., Wu, W., Zheng, Y., Lin, L., et al. (2019). Nitrogendoped Carbon Quantum Dots as an Antimicrobial Agent against Staphylococcus for the Treatment of Infected Wounds. Colloids Surf. B: Biointerfaces 179, 17-27. doi:10.1016/j.colsurfb.2019.03.042

Zhao, C., Wu, L., Wang, X., Weng, S., Ruan, Z., Liu, Q., et al. (2020). Quaternary Ammonium Carbon Quantum Dots as an Antimicrobial Agent against Gram-Positive Bacteria for the Treatment of MRSA-Infected Pneumonia in Mice.

Zhao, H. X., Liu, L. Q., Liu, Z. D., Wang, Y., Zhao, X. J., and Huang, C. Z. (2011). Highly Selective Detection of Phosphate in Very Complicated Matrixes with an Off-On Fluorescent Probe of EuropiumAdjusted Carbon Dots. Chem. Commun. 47 (9), 2604-2606. doi:10.1039/ c0cc04399k 
Zhao, L., Di, F., Wang, D., Guo, L.-H., Yang, Y., Wan, B., et al. (2013). Chemiluminescence of Carbon Dots under strong Alkaline Solutions: a Novel Insight into Carbon Dot Optical Properties. Nanoscale 5 (7), 2655-2658. doi:10.1039/c3nr00358b

Zhao, Q.-L., Zhang, Z.-L., Huang, B.-H., Peng, J., Zhang, M., and Pang, D.-W. (2008). Facile Preparation of Low Cytotoxicity Fluorescent Carbon Nanocrystals by Electrooxidation of Graphite. Chem. Commun. 7, 5116-5118. doi:10.1039/b812420e

Zheng, L., Chi, Y., Dong, Y., Lin, J., and Wang, B. (2009). Electrochemiluminescence of Water-Soluble Carbon Nanocrystals Released Electrochemically from Graphite. J. Am. Chem. Soc. 131 (13), 4564-4565. doi:10.1021/ja809073f

Zheng, X. T., Ananthanarayanan, A., Luo, K. Q., and Chen, P. (2015). Glowing Graphene Quantum Dots and Carbon Dots: Properties, Syntheses, and Biological Applications. Small 11 (14), 1620-1636. doi:10.1002/smll.201402648

Zhou, L., Chen, Z., Dong, K., Yin, M., Ren, J., and Qu, X. (2014). DNA-mediated Biomineralization of Rare-Earth Nanoparticles for Simultaneous Imaging and Stimuli-Responsive Drug Delivery. Biomaterials 35 (30), 8694-8702. doi:10.1016/j.biomaterials.2014.06.034

Zhu, A., Qu, Q., Shao, X., Kong, B., and Tian, Y. (2012). Carbon-Dot-Based DualEmission Nanohybrid Produces a Ratiometric Fluorescent Sensor for In Vivo Imaging of Cellular Copper Ions. Angew. Chem. 124 (29), 7297-7301. doi:10.1002/ange.201109089
Zhu, S., Meng, Q., Wang, L., Zhang, J., Song, Y., Jin, H., et al. (2013). Highly Photoluminescent Carbon Dots for Multicolor Patterning, Sensors, and Bioimaging. Angew. Chem. 125 (14), 4045-4049. doi:10.1002/ange.201300519

Conflict of Interest: The authors declare that the research was conducted in the absence of any commercial or financial relationships that could be construed as a potential conflict of interest.

Publisher's Note: All claims expressed in this article are solely those of the authors and do not necessarily represent those of their affiliated organizations, or those of the publisher, the editors, and the reviewers. Any product that may be evaluated in this article, or claim that may be made by its manufacturer, is not guaranteed or endorsed by the publisher.

Copyright (c) 2021 Azam, Najabat Ali and Javaid Khan. This is an open-access article distributed under the terms of the Creative Commons Attribution License (CC BY). The use, distribution or reproduction in other forums is permitted, provided the original author(s) and the copyright owner(s) are credited and that the original publication in this journal is cited, in accordance with accepted academic practice. No use, distribution or reproduction is permitted which does not comply with these terms. 